\title{
THE KINEMATIC FORMULA IN COMPLEX INTEGRAL GEOMETRY
}

BY

\author{
THEODORE SHIFRIN
}

\begin{abstract}
Given two nonsingular projective algebraic varieties $X, Y \subset P^{n}$ meeting transversely, it is classical that one may express the Chern classes of their intersection $X \cap Y$ in terms of the Chern classes of $X$ and $Y$ and the Kähler form (hyperplane class) of $\mathbf{P}^{n}$. This depends on global considerations. However, by putting a hermitian connection on the tangent bundle of $X$, we may interpret the Chern classes of $X$ as invariant polynomials in the curvature form of the connection. Armed with this local formulation of Chern classes, we now consider two complex submanifolds (not necessarily compact) $X, Y \subset \mathbf{P}^{n}$, and investigate the geometry of their intersection. The pointwise relation between the Chern forms of $X \cap Y$ and those of $X$ and $Y$ is rather complicated. However, when we average integrals of Chern forms of $X \cap \mathrm{g} Y$ over all elements $g$ of the group of motions of $\mathbf{P}^{n}$, these can be expressed in a universal fashion in terms of integrals of Chern forms of $X$ and $Y$. This is, then, the kinematic formula for the unitary group.
\end{abstract}

0. Introduction. We are interested in intrinsic integro-geometric invariants of complex submanifolds $X$ of $\mathbf{P}^{n}$, i.e., curvature integrals of the type $I_{j}(X)=\int_{X} c_{j}\left(\Omega_{X}\right)$ $\wedge \omega^{p-j}$, where

$$
c_{j}(\Omega)=\left(\frac{\sqrt{-1}}{2 \pi}\right)^{j} \frac{1}{j !} \sum \varepsilon_{\beta_{1} \ldots \beta_{j}}^{\alpha_{1} \ldots \alpha_{\alpha_{1}} \Omega_{1}} \wedge \cdots \wedge \Omega_{\alpha_{j} \bar{\beta}_{j}}
$$

is the $j$ th Chern form of $X^{p}$ and $\omega$ is the induced Kähler form. These invariants enter, for example, as coefficients in the formulas for the volume of a tube about $X \subset \mathbf{P}^{n}$ (cf. [12], [9]), analogous to Hermann Weyl's formulas in the real case (cf. [29], [12]). Now, given two submanifolds $X, Y \subset \mathbf{P}^{n}$ meeting transversely, it is natural to ask whether there is some relation among the various invariants $I_{j}(X \cap Y), I_{k}(X), I_{l}(Y)$. In the case that $X$ and $Y$ are nonsingular algebraic varieties, i.e., compact manifolds without boundary, it is classical that

$$
I_{j}(X \cap Y)=\sum_{0<k+l<j}(-1)^{j-k-l}\left(\begin{array}{c}
n+j-k-l \\
n
\end{array}\right) I_{k}(X) I_{l}(Y) .
$$

However, in the noncompact case, the situation resembles the real setting, in that the second fundamental forms of $X \cap Y$ in $X$ and in $Y$ appear. Chern considered in [6] a corresponding family of curvature invariants for a compact submanifold of $\mathbf{R}^{n}$-those which enter in Weyl's formulas-and proved a kinematic formula for

Received by the editors July 2, 1979 and, in revised form, February 26, 1980.

1980 Mathematics Subject Classification. Primary 53B05, 53B21, 53B35, 53B65; Secondary 14F05, $14 \mathrm{M} 15$.

Key words and phrases. Hermitian geometry, connections, curvature, Chern classes, Grassmannians, vector bundles, kinematic formula. 
Euclidean space. Similarly, a generalized version of $(0.1)$ holds "on the average", i.e., when we integrate over the group $U(n+1)$ of all motions of $\mathbf{P}^{n}$ :

$$
\begin{aligned}
\int_{U(n+1)} I_{j}(X & \cap g Y) d g \\
& =\operatorname{vol}(U(n+1)) \sum_{0<k+l<j}(-1)^{j-k-l}\left(\begin{array}{c}
n+j-k-l \\
n
\end{array}\right) I_{k}(X) I_{l}(Y) .
\end{aligned}
$$

This is the kinematic formula for $\mathbf{P}^{n}$ and constitutes the main result of this paper. In fact, we obtain a slightly stronger result for free.

(0.3) TheOREM. Let $P\left(\Omega^{\prime \prime}, \omega^{\prime \prime}\right)$ be a polynomial of total degree $2(p+q-n)$ in Chern forms $c_{i}\left(\Omega^{\prime \prime}\right)$ and the Kähler form $\omega^{\prime \prime}$. Then there is canonically associated to $P$ a polynomial $Q\left(\Omega, \Omega^{\prime}, \omega, \omega^{\prime}\right)$ of total degree $2(p+q)$ so that

$$
\int_{U(n+1)}\left(\int_{X \cap g Y} P\left(\Omega_{X \cap g Y}, \omega\right)\right) d g=\int_{X \times Y} Q\left(\Omega_{X}, \Omega_{Y}, \omega_{X}, \omega_{Y}\right)
$$

for any submanifolds $X^{p}, Y^{q} \subset \mathbf{P}^{n}$ for which both sides make sense.

On one level, $(0.2)$ is a direct generalization to the hermitian setting of Chern's theorem in [6]. In the real case, except for the Gauss-Bonnet integral, none of the integro-geometric invariants is topological in character, whereas, in the complex case, all the integrands $c_{j}\left(\Omega_{X}\right) \wedge \omega^{p-j}$ represent cohomology classes on $X$. That the intrinsic curvature invariants of a hermitian manifold are all expressible in terms of the Chern classes is essentially a consequence of elementary invariant theory, or, equivalently, of the topology of complex Grassmannians. This, then, is the crucial link between the differential geometry and the algebro-geometric formula (0.1).

Our work is based on the theory of moving frames. We begin then with a review of standard results in hermitian geometry with emphasis on this point of view. We omit most proofs and refer the reader to [7] and [14] for background. We include a brief discussion of the cohomology of homogeneous and symmetric spaces, as this plays a key rôle in our later work. Some technicalities on the geometry of integration over the fiber are included in an appendix, along with some detailed applications of Weyl's first main theorem on vector invariants (1.27) which prove crucial.

We turn in $\$ 2$ to some elementary theorems in complex integral geometry, results we refer to as Crofton formulas, since they formally resemble the original work of Crofton in geometric probability. This lays the groundwork for our main results. We begin in $\$ 3$ by proving a special case of $(0.1)$ and $(0.2)$, namely the case $Y=\mathbf{P}^{q}$, which Chern refers to as the reproductive property of the curvature integrals $I_{j}(X)$.

The proof is, however, unlike Chern's in that we now exploit the topology of the situation. The main geometric point is that intrinsically associated to the submanifold $X \subset \mathbf{P}^{n}$ is the "pointed Gauss mapping"

$$
\begin{gathered}
\gamma: X \rightarrow \mathbf{G}(0, p, n)=\left\{(z, T) \in \mathbf{P}^{n} \times \mathbf{G}(p, n): z \in T\right\}, \\
\gamma(z)=\left(z, \widetilde{T_{z} X}\right),
\end{gathered}
$$


where $\widetilde{T_{z} X}$ is the projective $p$-plane tangent to $X$ at $z$. By means of this mapping, we are able to reduce the problem at hand to one of determining the invariant forms on the flag space $\mathbf{G}(0, p, n)$. Here we encounter the problem that while $\mathbf{G}(0, p, n)$ is a homogeneous space, it is not a hermitian symmetric space and thus admits too many invariant forms. We are able, however, to discard all but the closed forms in the context of our work here, and then matters are quite manageable.

Lastly, in $\$ 4$ we prove the general formula $(0.2)$. Here we must determine the invariant forms on the product $\mathbf{G}(0, p, n) \times \mathbf{G}(0, q, n)$; this process is unwieldy since the space is no longer even homogeneous. So we must resort to explicit moving frames computations in order to isolate the group's actions on each of the two spaces; here we introduce the formalism of Stiefel bundles on the Grassmannians. The arguments then reduce to those made in the linear case. We conclude with a computation which ascertains convergence near points of nontransverse intersection.

We expect this theory to prove useful in studying varieties with singularities. Since curvature forms in the complex analytic setting have a definite sign, the study of convergence of integrals is greatly simplified (cf. [12]), and we expect some fruitful applications of these kinematic formulas to Plücker-type formulas in higher dimensions and to working with Chern classes of singular varieties (cf. [20]). Similar sorts of methods have already proved valuable in deriving some integrogeometric formulas regarding polar varieties associated to foliations [19]. Moreover, we plan to study similar kinematic formulas for geometric invariants which are not topological, thereby utilizing the full structure of the ring of invariant forms.

\section{Review of hermitian geometry.}

(a) Moving frames. Let $\mathbf{C}^{n}$ denote $n$-dimensional complex space with coordinates $z=\left(z^{1}, \ldots, z^{n}\right)$ and hermitian inner product $(z, w)=\sum z^{i} w^{i}$. Complex projective $n$-space, $\mathbf{P}^{n}$, inherits from the natural projection $\pi: \mathbf{C}^{n+1}-\{0\} \rightarrow \mathbf{P}^{n}$ a complex structure and a hermitian structure. Letting $z^{0}, \ldots, z^{n}$ be homogeneous coordinates on $\mathbf{P}^{n}$, the unique hermitian metric which is invariant under the natural action of the unitary group $U(n+1)$ is

$$
d s^{2}=\sum \frac{\partial^{2} \log \|z\|^{2}}{\partial z^{i} \partial z^{\bar{j}}} d z^{i} \otimes d z^{\bar{j}},
$$

and the associated Kähler form is $\omega=(\sqrt{-1} / 2 \pi) \partial \partial \overline{l o g}\|z\|^{2}$. Here we have included the normalizing factor of $1 / \pi$ so that $\omega$ represents the (positive) generator of the integral cohomology $H^{2}\left(\mathbf{P}^{n}, \mathbf{Z}\right)$, i.e., the Poincare dual of a hyperplane.

One of the distinguishing features of hermitian geometry is the following fact.

(1.1) Lemma. If $X$ is a Kähler manifold with Kähler form $\omega$, and $Z \subset X$ is a $k$-dimensional complex submanifold, then

(a) $Z$ is a Kähler manifold with Kähler form $\left.\omega\right|_{Z}$; and

(b) $\omega^{k} / k$ ! gives the volume form for $Z$ in the induced hermitian metric.

From this it follows, for example, that the degree of an algebraic subvariety $X^{k} \subset \mathbf{P}^{n}$ is its most primitive geometric invariant: the degree is the number of 
points of intersection of $X$ with a generic $\mathbf{P}^{n-k}$; by Poincaré duality (cf. (A.4)), this number is $\int_{X} \omega^{k}=\left(k ! / \pi^{k}\right) \operatorname{vol}(X)$.

The most important tool in our work will be the calculus of moving frames. A moving frame on an open set $U \subset C^{n}$ is a set $\left(e_{1}(z), \ldots, e_{n}(z)\right)$ of smooth $\mathbf{C}^{n}$-valued functions such that for each $z \in U,\left(e_{1}, \ldots, e_{n}\right)$ is a unitary frame for $T_{z} \mathbf{C}^{n} \cong \mathbf{C}^{n}$. Let $z: U \hookrightarrow \mathbf{C}^{n}$ be the inclusion mapping, so that the vector-valued 1 -form $d z$ is the identity at each point of $U$. Given a moving frame $e_{1}, \ldots, e_{n}$ on $U$, define $\omega^{i}=\left(d z, e_{i}\right), \omega_{i j}=\left(d e_{i}, e_{j}\right)$, where $(\cdot, \cdot)$ denotes the hermitian inner product on $\mathbf{C}^{n}$; i.e., by this we mean

$$
\left\{\begin{array}{l}
d z=\sum \omega^{i} \otimes e_{i}, \\
d e_{i}=\sum \omega_{i j} \otimes e_{j} .
\end{array}\right.
$$

Note that $\omega^{i}, \omega^{i}$ give a basis for all the l-forms on $U$ (since $\omega^{i}$ is nothing but the dual of $\left.e_{i}\right)$. Since $\left(e_{i}, e_{j}\right)=\delta_{i j}$, we obtain

$$
\omega_{i j}+\bar{\omega}_{j i}=0
$$

The crucial formulas relating all these forms are the so-called structure equations of $\mathbf{C}^{n}$ (here we let $1 \leqslant i, j, k \leqslant n$ ):

$$
d \omega^{i}=\sum \omega^{j} \wedge \omega_{j i}, \quad d \omega_{i \bar{j}}=\sum \omega_{i \bar{k}} \wedge \omega_{k \bar{j}}
$$

Now, let $X^{p} \subset \mathbf{C}^{n}$ be a complex submanifold. A moving frame on $U \subset X$ consists of smooth $\mathrm{C}^{n}$-valued functions $\left(e_{1}, \ldots, e_{p}, e_{p+1}, \ldots, e_{n}\right)$ such that for each $z \in U, e_{1}, \ldots, e_{p}$ span $T_{z} X \cong \mathbf{C}^{p}$ and $e_{1}, \ldots, e_{n}$ are a unitary frame for $T_{z} \mathbf{C}^{n} \cong \mathbf{C}^{n}$. As before, we introduce 1 -forms $\omega^{i}$ and $\omega_{i j}$, and we observe that $\omega^{p+1}=\cdots=\omega^{n}=0$ on $X$ since $e_{p+1}, \ldots, e_{n}$ are normal to $X$. We now introduce the index ranges

$$
1 \leqslant \alpha, \beta, \gamma \leqslant p, \quad p+1 \leqslant \mu, \nu \leqslant n, \quad 1<i, j, k<n,
$$

and notice that the structure equations can be rewritten, slightly modified, as follows.

Proposition (Structure eQuations for a submanifold of $\mathbf{C}^{\boldsymbol{n}}$ ).

$$
\begin{aligned}
& d \omega^{i}=\sum \omega^{\alpha} \wedge \omega_{\alpha \bar{i}} \\
& d \omega_{i \bar{j}}=\sum \omega_{i \bar{k}} \wedge \omega_{k \bar{j}}
\end{aligned}
$$

We define the curvature forms $\Omega_{\alpha \bar{\beta}}$ of $X$ by

$$
\Omega_{\alpha \bar{\beta}}=d \omega_{\alpha \bar{\beta}}-\sum \omega_{\alpha \bar{\gamma}} \wedge \omega_{\gamma \bar{\beta}} .
$$

Using (1.2) one checks that $\Omega_{\alpha \bar{\beta}}+\bar{\Omega}_{\beta \bar{\alpha}}=0$, and it follows from the structure equations (1.5) and from (1.6) that

$$
\Omega_{\alpha \bar{\beta}}=\sum \omega_{\alpha \bar{\mu}} \wedge \omega_{\mu \bar{\beta}}=-\sum \omega_{\alpha \bar{\mu}} \wedge \bar{\omega}_{\beta \bar{\mu}} .
$$

We would like next to consider moving frames on a submanifold $X$ of $\mathbf{P}^{n}$; here the concept is essentially that of a projective connection on $X$ (cf. [8]). The essential point is that for submanifolds of projective space, the relation between intrinsic 
and extrinsic geometry becomes somewhat more involved. One must, in particular, distinguish between the abstract tangent bundle and the "projective tangent bundle", and it is to this task that we now turn.

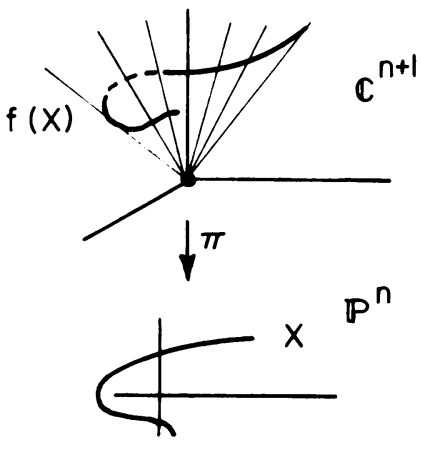

FIGURE 1

Let $X^{p} \subset \mathbf{P}^{n}$ be a complex submanifold. First we must define the tangent projective $p$-plane $\widetilde{T_{z} X}$ to $X$ at $z$. Naïvely, it is the projective $p$-plane which osculates $X$ to first order at $z$. Let $f$ be a holomorphic lifting of $X$ to $\mathbf{C}^{n+1}$ in a neighborhood $U$ of $z$. Consider the $(p+1)$-plane $\tilde{E}_{z}$ spanned by $f(z)$ and $f_{*}\left(T_{z} X\right)$ in $\mathrm{C}^{n+1}$; define $\widetilde{T_{z} X}=\pi\left(\tilde{E}_{z}\right)$. Geometrically, we are choosing a local section of the cone $\pi^{-1}(X)$ and $\tilde{E}_{z}$ is the tangent plane to the cone at the point lying over $z$. (See Figure 1.) One checks easily that $\widetilde{T_{z} X}$ is well defined. Moreover, these linear spaces $\tilde{E}_{z}$ fit together smoothly to form a holomorphic vector bundle $\tilde{E}$ of $\operatorname{rank}(p+1)$ on $X$. Then we have the natural

Definition. The projective tangent bundle $\widetilde{T X}$ of $X$ is defined to be $\mathbf{P}(\tilde{E})$, i.e., the holomorphic fiber bundle whose fiber over $z \in X$ is the projective $p$-plane $\pi\left(\tilde{E}_{z}\right)=$ $\mathbf{P}\left(\tilde{E}_{z}\right)$.

It is now an easy matter to define (adapted unitary) moving frames on $X \subset \mathbf{P}^{n}$. A moving frame (see Figure 2) on $U \subset X$ consists of $n+1 C^{n+1}$-valued smooth functions $e_{0}, \ldots, e_{n}$ on $U$ such that

(i) $\pi\left(e_{0}\right)=z$,

(ii) $e_{0}, \ldots, e_{p} \operatorname{span} \tilde{E}_{z}$,

(iii) $e_{0}, \ldots, e_{n}$ are a unitary frame.

As before, one then defines differential forms $\omega_{A \bar{B}}=\left(d e_{A}, e_{B}\right), 0<A, B<n$, on $U$. We shall return to the structure equations shortly.

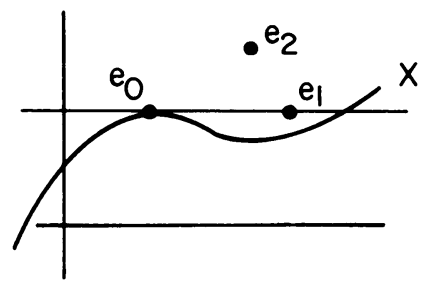

FIGURE 2 
(b) Connections and Chern classes. We now give a brief summary of the theory of connections on complex vector bundles in order to recall the differential-geometric definition of Chern classes. For more details, we refer the reader to [7], [14], [28]. Let $X$ be a complex manifold and let $\pi: E \rightarrow X$ be a smooth complex vector bundle of rank $r$. A hermitian metric on $E$ is a smooth assignment of hermitian metrics $(\cdot, \cdot)_{z}$ in each fiber $\pi^{-1}(z)$; it is easy to construct a hermitian metric on any vector bundle using partitions of unity. Given a choice $s=\left(s_{1}, \ldots, s_{r}\right)$ of local frame field for $E$, we define $h_{i j}(z)=\left(s_{i}(z), s_{j}(z)\right)_{z}$; the $h_{i j}$ are smooth functions depending on $s$. We say the frame field is unitary if $h_{i j}=\delta_{i j}$. Note that a hermitian metric on $T X$ is identical to a hermitian metric on $X$. We say $E \stackrel{\pi}{\rightarrow} X$ is a holomorphic vector bundle if $E$ is a complex manifold and $\pi$ is holomorphic; we say $E$ is a hermitian vector bundle if it is a holomorphic vector bundle with a hermitian metric.

A connection on a vector bundle gives a means of differentiating sections and its associated curvature measures the extent to which derivatives fail to commute. Specifically, let $\Gamma(E)$ denote global complex-valued $C^{\infty}$ sections and $T^{*} X$ the complexified cotangent bundle. A connection $D$ on $E$ is a $C$-linear mapping $D$ : $\Gamma(E) \rightarrow \Gamma\left(T^{*} X \otimes E\right)$ such that $D(f s)=d f \otimes s+f D s$ for $s \in \Gamma(E), f \in C^{\infty}$. If $s=\left(s_{1}, \ldots, s_{r}\right)$ is a local frame field on an open set $U \subset X$, we define 1-forms $\omega_{i}^{j}$ (here $1 \leqslant i, j, k \leqslant r$ ) on $U$ by $D s_{i}=\sum \omega_{i}^{j} \otimes s_{j}$ and say that $\left(\omega_{i}^{j}\right)$ is the connection matrix relative to the frame $s$. As a matter of notation, we agree to lower the index and write $\omega_{i j}$ for the connection matrix with respect to a unitary frame.

Thus, if $\sigma$ is an arbitrary section, we put $\sigma=\sum a^{i} s_{i}$, and $D \sigma=\Sigma\left(d a^{j}+a^{i} \omega_{i}^{j}\right) \otimes$ $s_{j}$. We then extend the action of $D$ to $\Lambda^{p} T^{*} X \otimes E$ as follows.

$$
\begin{gathered}
D: \Gamma\left(\Lambda^{p} T^{*} X \otimes E\right) \rightarrow \Gamma\left(\Lambda^{p+1} T^{*} X \otimes E\right), \\
D\left(\phi \otimes s_{i}\right)=d \phi \otimes s_{i}+(-1)^{p} \sum\left(\phi \wedge \omega_{i}^{j}\right) \otimes s_{j} .
\end{gathered}
$$

We now compute

$$
D\left(D s_{i}\right)=D\left(\sum \omega_{i}^{j} \otimes s_{j}\right)=\sum\left(d \omega_{i}^{j}-\sum \omega_{i}^{k} \wedge \omega_{k}^{j}\right) \otimes s_{j} .
$$

Setting $D\left(D s_{i}\right)=\Sigma \Omega_{i}^{j} \otimes s_{j}$ defines a matrix $\left(\Omega_{i}^{j}\right)$ of 2-forms, which we term the curvature matrix of $D$ with respect to the frame field $s$. In matrix notation, we have $\Omega=d \omega-\omega \wedge \omega$.

One of the central formulas emerges when we consider how the connection and curvature matrices transform when we change frame fields.

LEMma. Let $g: U \rightarrow \mathrm{Gl}(r, \mathrm{C})$ be a smooth change of frame field; i.e., define $s_{i}^{\prime}(z)=\sum g_{i j}(z) s_{j}(z)$. Then

$$
\omega^{\prime}=g \omega g^{-1}+d g \cdot g^{-1}, \quad \Omega^{\prime}=g \Omega g^{-1} .
$$

The proof is straightforward computation and is omitted. Cf. [7], [14].

In the case of real vector bundles, it is essentially only for the tangent bundle that one can find a unique connection compatible with the Riemannian metric (the Levi-Civita connection). However, in the case of holomorphic vector bundles on complex manifolds, the underlying complex structure provides a preferred connection. Since any differential form on a complex manifold can be decomposed into 
forms of type $(p, q)$, we may consider the $(0,1)$ component of the connection $D$. In particular, we say $D$ is compatible with the complex structure if the $(0,1)$ component of $D$ is $\bar{\partial}$; on the other hand, we say $D$ is compatible with the hermitian structure if, for any two sections $\sigma, \sigma^{\prime}, d\left(\sigma, \sigma^{\prime}\right)=\left(D \sigma, \sigma^{\prime}\right)+\left(\sigma, D \sigma^{\prime}\right)$. We now have the

Proposition. Let $E \rightarrow X$ be a hermitian vector bundle. There is a unique connection $D$ on $E$ compatible with both the holomorphic structure and the hermitian structure. We call the connection so obtained the canonical connection on $E$.

Suppose we now compute with respect to a unitary frame field $s$. Then we have the important

Proposition. The curvature matrix of the canonical connection with respect to a unitary frame field is a skew-hermitian matrix of forms of type $(1,1)$.

We turn next to a brief discussion of Chern classes of hermitian vector bundles. While there is a plethora of definitions, the usual ones (e.g., classifying spaces, splitting principle, obstruction theory) depend on global topological considerations and give the Chern classes as cohomology classes. Since we are ultimately interested in local phenomena, we need a specific cocycle representing the cohomology class, and it is most convenient to use differential forms. This was essentially Chern's definition of Chern classes in [3].

Let $E \rightarrow X$ be a complex vector bundle of rank $r$ over a complex manifold $X$. Define a connection $D$ (arbitrarily) on $E$ and let $\Omega$ be its curvature matrix with respect to a given frame field. Define forms $c_{k}(\Omega)$ by

$$
\operatorname{det}\left(t I+\frac{\sqrt{-1}}{2 \pi} \Omega\right)=\sum_{k=0}^{r} c_{k}(\Omega) t^{r-k} .
$$

While a priori these forms are only locally defined and depend on the choice of frame field, note that the left-hand side is invariant under conjugation by $\mathrm{Gl}(r, \mathrm{C})$ and since $\Omega$ transforms by (1.8) under change of frame, the forms $c_{k}(\Omega)$ are in fact globally defined. It is a direct computation to check that

$$
c_{j}(\Omega)=\left(\frac{\sqrt{-1}}{2 \pi}\right)^{j} \frac{1}{j !} \sum \varepsilon_{\beta_{1} \cdots \beta_{j}}^{\alpha_{1} \cdots \alpha_{\alpha_{1}}^{\beta_{1}}} \wedge \cdots \wedge \Omega_{\alpha_{j}}^{\beta_{j}}
$$

where $\varepsilon_{\beta_{1} \cdots \beta_{j}}^{\alpha_{1} \cdots \alpha_{j}}$ is the sign of the permutation $\left(\alpha_{1}, \ldots, \alpha_{j}\right) \leadsto\left(\beta_{1}, \ldots, \beta_{j}\right)$. For example, we have

$$
c_{1}(\Omega)=(\sqrt{-1} / 2 \pi) \operatorname{tr}(\Omega), \quad c_{r}(\Omega)=(\sqrt{-1} / 2 \pi)^{r} \operatorname{det}(\Omega) .
$$

It follows immediately from the Bianchi identity that $c_{k}(\Omega)$ are closed forms (cf. [17]).

If we take $E$ to be a hermitian vector bundle and equip it with the canonical connection, we then compute the curvature matrix $\Omega_{E}$ with respect to a unitary frame field. Then since $\Omega_{E}$ is a skew-hermitian matrix of $(1,1)$ forms, one checks easily that

$$
c_{k}\left(\Omega_{E}\right)=\left(\frac{\sqrt{-1}}{2 \pi}\right)^{k} \frac{1}{k !} \sum \varepsilon_{\beta_{1} \cdots \beta_{k}}^{\alpha_{1} \cdots \alpha_{k}} \Omega_{\alpha_{1} \bar{\beta}_{1}} \wedge \cdots \wedge \Omega_{\alpha_{k} \bar{\beta}_{k}}
$$


is a closed, real $(k, k)$ form on $X$, thereby defining a cohomology class in $H^{k, k}(X) \cap H^{2 k}(X, \mathbf{R}) \subset H^{2 k}(X, \mathbf{C})$. We shall refer to these forms as the Chern forms of $E$. When we think of the cohomology classes defined by the forms, we shall of ten write $\left[c_{k}\left(\Omega_{E}\right)\right]=c_{k}(E)$.

The assorted standard properties of Chern classes hold on the level of forms, and we list them here. Define the total Chern form $c\left(\Omega_{E}\right)=1+c_{1}\left(\Omega_{E}\right)+\cdots+c_{r}\left(\Omega_{E}\right)$. Unless we specify otherwise, by $\Omega_{E}$ we shall always mean the curvature matrix of the canonical connection with respect to a unitary frame field.

(1.10) THEOREM. (1) If $f: Y \rightarrow X$ is a holomorphic mapping of complex manifolds and $E$ is a hermitian vector bundle on $X$, then

$$
c\left(\Omega_{f^{*} E}\right)=f^{*} c\left(\Omega_{E}\right) \quad \text { (naturality). }
$$

(2) Let $0 \rightarrow E^{\prime} \rightarrow E \rightarrow E^{\prime \prime} \rightarrow 0$ be a short exact sequence of hermitian vector bundles on $X$. Then

$$
c\left(\Omega_{E}\right)=c\left(\Omega_{E^{\prime}}\right) \wedge c\left(\Omega_{E^{\prime \prime}}\right)
$$

(3) Let $E$ be a hermitian vector bundle of rank $r, L$ a hermitian line bundle. Then

$$
c_{k}\left(\Omega_{E \otimes L}\right)=\sum_{l=0}^{k}\left(\begin{array}{c}
r-l \\
k-l
\end{array}\right) c_{l}\left(\Omega_{E}\right) \wedge c_{1}\left(\Omega_{L}\right)^{k-l} .
$$

We return to a submanifold $X \subset \mathbf{P}^{n}$. In order to compare the projective tangent bundle $\overparen{T X}$ and the abstract tangent bundle $T X$, we recall the definition of the tautological or universal line bundle $H^{-1}$ on $\mathbf{P}^{n}$. Viewing $\mathbf{P}^{n}$ as lines through the origin in $\mathbf{C}^{n+1}$, we define

$$
H^{-1}=\left\{(l, Z) \in \mathbf{P}^{n} \times \mathbf{C}^{n+1}: Z \in l\right\}
$$

i.e., the fiber over a point in $\mathbf{P}^{n}$ is the line in $\mathbf{C}^{n+1}$ represented by the point. It is easy to check that $\|Z\|^{2}$ defines a hermitian metric on $H^{-1}$ (thinking of $Z$ as homogeneous coordinates on $\mathbf{P}^{n}$ ); the associated connection form is given by $\partial \log \|Z\|^{2}$, and the curvature form by $\Omega=\bar{\partial} \partial \log \|Z\|^{2}$. One checks that $c_{1}\left(\Omega_{H^{-1}}\right)=$ $(\sqrt{-1} / 2 \pi) \Omega$ represents $-1 \in H^{2}\left(\mathbf{P}^{n}, \mathbf{Z}\right) \cong \mathbf{Z}$ with a simple integration. (The dual bundle $H$ is called the hyperplane bundle and has Chern class +1 ; its Chern form is $(\sqrt{-1} / 2 \pi) \partial \bar{\partial} \log \|Z\|^{2}=\omega=$ Kähler form of $\left.\mathbf{P}^{n}.\right)$

Then it is natural to view $T \mathbf{P}^{n}$ as $\operatorname{Hom}\left(H^{-1}, \varepsilon^{n+1} / H^{-1}\right)$, where $\varepsilon^{n+1} / H^{-1} \cong$ $\left(H^{-1}\right)^{\perp}$ is the orthogonal complement with respect to the hermitian metric on the trivial $(n+1)$-plane bundle $\varepsilon^{n+1}$ (cf. [21, pp. 169-170]). From the point of view of moving frames, we are saying that for a choice of unitary frame field $e_{0}, \ldots, e_{n}$ in a neighborhood of $z=\pi\left(e_{0}\right)$, the $(1,0)$ cotangent space $T_{z}^{(1,0)} \mathbf{P}^{n}$ is spanned by $\omega_{0 \overline{1}}, \ldots, \omega_{0 \bar{n}}$. Similarly, given a submanifold $X$ of $\mathbf{P}^{n}$, we would expect $\omega_{0 \overline{1}}, \ldots, \omega_{0 \bar{p}}$ to $\operatorname{span} T^{(1,0)} X$. Geometrically, this is saying that $T X \simeq$ $\operatorname{Hom}\left(H^{-1}, \tilde{E} / H^{-1}\right)=H \otimes\left(\tilde{E} / H^{-1}\right)=(\tilde{E} \otimes H) / \varepsilon$. In fact, this holds in the holomorphic category as well.

(1.11) Proposition (Euler sequence).

$$
0 \rightarrow H^{-1} \rightarrow \tilde{E} \rightarrow T X \otimes H^{-1} \rightarrow 0
$$

is an exact sequence of hermitian vector bundles on $X$, where by $H^{-1}$ we mean the restriction of the tautological line bundle to $X$. 
REMARK. On one hand, $H^{-1} \hookrightarrow \tilde{E}$, since the tautological line is clearly a subspace of each fiber of $\tilde{E}$. On the other hand, $\pi_{*}$ seems to give a mapping $\tilde{E} \rightarrow T X$, but this only works locally, since any choice of section of $\tilde{E}$ is naturally twisted by $H^{-1}$ (i.e., by the local lifting $f$ ). Thus, it is not surprising that the twist appears in the sequence.

A proof of Proposition (1.11) can be given along the lines of [14, pp. 408-409] or by a direct computation of transition functions.

The Euler sequence gives a specific relation between the Chern forms of $X$ and those of the vector bundle $\tilde{E}$. In particular, the exact sequence (1.11) gives

$$
0 \rightarrow \varepsilon^{1} \rightarrow \tilde{E} \otimes H \rightarrow T X \rightarrow 0,
$$

and thus by $(1.10), c\left(\Omega_{X}\right)=c\left(\Omega_{\tilde{E} \otimes H}\right)$. We conclude

(1.12) Corollary. $c\left(\Omega_{\mathbf{p}^{n}}\right)=(1+\omega)^{n+1}$.

(1.13) CoROLlaRY. $c_{k}\left(\Omega_{X}\right)=\sum_{l=0}^{k}\left(\begin{array}{c}p+1-l \\ k-l\end{array}\right) c_{l}\left(\Omega_{\tilde{E}}\right) \wedge \omega^{k-l}$, and, conversely,

$$
c_{k}\left(\Omega_{\tilde{E}}\right)=\sum_{l=0}^{k}(-1)^{k-l}\left(\begin{array}{c}
p+1-l \\
k-l
\end{array}\right) c_{l}\left(\Omega_{X}\right) \wedge \omega^{k-l} .
$$

(c) Grassmannians. One can view the Grassmannian $G(p, n)$ of $p$-dimensional subspaces of $\mathbf{C}^{n}$ as a homogeneous space in either of two ways. First of all, $G(p, n)=U(n) /(U(p) \times U(n-p))$ and hence is compact; this approach will be important in considering unitary frames and coframes on the Grassmannian. On the other hand, if we let $K \subset \mathrm{Gl}(n, \mathrm{C})$ denote the subgroup consisting of matrices of the form

$$
\left[\begin{array}{c|c}
A & B \\
\hline 0 & C
\end{array}\right], \quad A \in \mathrm{Gl}(p, \mathbf{C}), B \text { arbitrary, } C \in \mathrm{Gl}(n-p, \mathbf{C}),
$$

then $G(p, n)=\mathrm{Gl}(n, \mathrm{C}) / K$, and hence is a complex manifold. As a matter of notation, define $\mathbf{G}(p, n)=G(p+1, n+1)=$ the space of projective $p$-planes in $\mathbf{P}^{n}$.

In order to discuss moving frames on Grassmannians, we must first consider the hermitian geometry of the unitary group $U(n)$. The rows of a matrix $g \in U(n)$ give a unitary frame $e_{1}, \ldots, e_{n}$ for $\mathbf{C}^{n}$. Since the $e_{i}$ are therefore smooth $\mathbf{C}^{n}$-valued functions on $U(n), d e_{i}$ are $C^{n}$-valued 1 -forms on $U(n)$ and we can define the 1-forms $\omega_{i j}=\left(d e_{i}, e_{j}\right)$ on $U(n)$ using the hermitian inner product on $\mathbf{C}^{n}$. Since $\left(e_{i}, e_{j}\right)=\delta_{i j}$, differentiation gives $\omega_{i j}+\bar{\omega}_{j i}=0$. Moreover, we find that these forms are the right-invariant Maurer-Cartan forms on $U(n)$; for putting $\omega=\left(\omega_{i j}\right)$, we see that

$$
\omega_{i \bar{j}}=\left(d e_{i}, e_{j}\right)=\sum_{k} d g_{i k} \bar{g}_{j k}=\left(d g \cdot g^{-1}\right)_{i \bar{j}},
$$

since $g \in U(n)$, and therefore we have $\omega=d g \cdot g^{-1}$. To see $\omega$ is right-invariant, then, we compute $R_{g_{0}}^{*} \omega=d\left(g g_{0}\right)\left(g g_{0}\right)^{-1}=d g \cdot g_{0} g_{0}^{-1} g^{-1}=\omega$, as required. Since $\left\{\omega_{i j}\right\}$ give a basis for the 1 -forms at the identity element of $U(n)$, by rightinvariance they give a parallelism of $T^{*} U(n)$. 
The structure equations of the unitary group are given by

(1.14) Proposition (Maurer-Cartan equations).

$$
d \omega_{i \bar{j}}=\sum \omega_{i \bar{k}} \wedge \omega_{k \bar{j}} ; \quad \text { i.e., } \quad d \omega=\omega \wedge \omega .
$$

Proof. Since $g g^{-1}=I, d\left(g^{-1}\right)=-g^{-1} \cdot d g \cdot g^{-1}$. Therefore, $d \omega=d\left(d g \cdot g^{-1}\right)=$ $-d g \wedge d\left(g^{-1}\right)=d g \wedge g^{-1} \cdot d g \cdot g^{-1}=\omega \wedge \omega$.

We now turn to the study of the Grassmannian as a homogeneous space. We define $G(p, n)$ as the space of right cosets but will abuse notation for typographical reasons by writing $G(p, n)=G / H$, where $G=U(n), H=U(p) \times U(n-p)$. The principal bundle

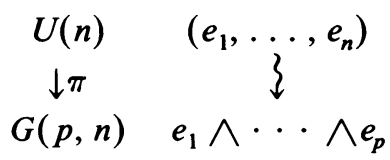

with group $H$ acting on the left is the bundle of adapted unitary frames on the Grassmannian, and we may use local sections of this bundle to pull the MaurerCartan forms of $G$ back to $G / H$.

(1.15) Proposition. The forms $\omega_{\alpha \bar{\mu}}, \bar{\omega}_{\alpha \bar{\mu}}$ on $U(n)$ are horizontal for the fibration $U(n) \stackrel{\pi}{\rightarrow} G(p, n)$, and for any local section $s, s^{*} \omega_{\alpha \bar{\mu}}$ are forms of type $(1,0)$ on $G(p, n)$ and hence are a basis for $T^{(1,0)} G(p, n)$.

Proof. (1) Let $e=$ identity in $G=U(n)$. Since the fiber over $H g \in G / H$ is the right translate by $g$ of the fiber over $H e$, and since the forms $\omega_{\alpha \bar{\mu}}$ are right-invariant, it suffices to verify that $\omega_{\alpha \bar{\mu}}(e)$ is horizontal. But a vertical vector $Y$ at $e$ is an element of the Lie algebra $\mathfrak{h}$, and $\omega_{\alpha \bar{\mu}}(e)(Y)$ is the $(\alpha, \mu)$ component of $Y$, which is zero by definition of $H$.

(2) That the forms $s^{*} \omega_{\alpha \bar{\mu}}$ are of type $(1,0)$ is not at all clear insofar as we are using unitary frames. The crucial point is to express $s^{*} \omega_{\alpha \bar{\mu}}$ in terms of a holomorphic frame field. Let $Z_{1}, \ldots, Z_{p}, Z_{p+1}, \ldots, Z_{n}$ be an adapted holomorphic frame field (i.e., $Z_{1}, \ldots, Z_{p}$ span the $p$-plane), or, in other words, a local holomorphic section of the bundle $\mathrm{Gl}(n, \mathrm{C}) \rightarrow \mathrm{Gl}(n, \mathrm{C}) / K \cong G(p, n)$.

If $s=\left(e_{1}, \ldots, e_{n}\right)$ is a local unitary frame field, then there are smooth functions $s_{\alpha \bar{\beta}}$ such that $e_{\alpha}=\sum s_{\alpha \bar{\beta}} Z_{\beta}$. Therefore,

$$
s^{*} \omega_{\alpha \bar{\mu}}=\left(d e_{\alpha}, e_{\mu}\right)=\sum s_{\alpha \bar{\beta}}\left(d Z_{\beta}, e_{\mu}\right)+\sum d s_{\alpha \bar{\beta}}\left(Z_{\beta}, e_{\mu}\right)
$$

the first term is of type $(1,0)$ and the second term vanishes.

While choosing a local section $s$ enables us to pull the forms back to $G / H$, these forms do not extend to globally defined forms on $G / H$. Clearly some sort of invariance under the action of $H$ is necessary for this to happen. We expand on this in the next few results.

(1.16) Proposition. Let $\pi: G \rightarrow G / H$. Given a $k$-form $\phi$ on $G$, there exists a form $\psi$ on $G / H$ such that $\phi=\pi^{*} \psi$, i.e., $\phi$ descends to a form $\psi$ on $G / H$, if and only if (i) $\phi$ is horizontal, and (ii) $L_{h}^{*} \phi=\phi$ for $h \in H$.

Cf. [17, Vol. 2, p. 294]. 
Now, so far as the Maurer-Cartan forms of $G$ are concerned, we have the

(1.17) Lemma. Let $U \subset G / H$ be an open set over which the bundle $G \rightarrow G / H$ is trivial, and let $s: U \rightarrow G$ be a section on $U$. Let $h: U \rightarrow H$ be a smooth function. Then

$$
(h s)^{*} \omega=h\left(s^{*} \omega\right) h^{-1}+d h \cdot h^{-1}
$$

where $\omega=\left(\omega_{i j}\right)$ is the matrix of Maurer-Cartan forms of $G$.

Proof. This is straightforward computation and is omitted.

One elementary consequence of Lemma (1.17) is the following fact which is a great aid in computations.

(1.19) Corollary. Given $x_{0} \in G / H$, there exists a local section s (i.e., an appropriate choice of local frame field) such that $s^{*}\left(\omega_{\alpha \bar{\beta}}\right)\left(x_{0}\right)=s^{*}\left(\omega_{\mu \bar{\nu}}\right)\left(x_{0}\right)=0$.

Proof. Let $U \subset G / H$ be a neighborhood of $x_{0}$ over which the bundle is trivial, and let $s: U \rightarrow G$ be an arbitrary section. Put $h\left(x_{0}\right)=I$,

$$
-d h\left(x_{0}\right)=\left(\begin{array}{c|c}
s^{*} \omega_{\alpha \bar{\beta}}\left(x_{0}\right) & 0 \\
\hline 0 & s^{*} \omega_{\mu \bar{\nu}}\left(x_{0}\right)
\end{array}\right) .
$$

Then it follows from (1.18) that $(h s)^{*} \omega_{\alpha \bar{\beta}}\left(x_{0}\right)=(h s)^{*} \omega_{\mu \bar{\nu}}\left(x_{0}\right)=0$.

REMARK. The fact that one may annihilate what is essentially a connection matrix at a fixed point by an appropriate choice of frame field is a generalization of Gauss normal coordinates in Riemannian geometry.

While the forms $\omega_{\alpha \bar{\mu}}$ are horizontal, we see from (1.18) that they are not left-invariant under $H$. Nevertheless, certain combinations will be and hence will descend by Proposition (1.16) to globally defined forms on $G / H$. For example, $\sum \omega_{\alpha \bar{\mu}} \otimes \bar{\omega}_{\alpha \bar{\mu}}$ and $\psi=(\sqrt{-1} / 2) \sum \omega_{\alpha \bar{\mu}} \wedge \bar{\omega}_{\alpha \bar{\mu}}$ are invariant and give the hermitian (Fubini-Study) metric and Kähler form of $G(p, n)$ respectively, as one can easily check. Granting that we know $\psi$ is a well-defined form on $G(p, n)$, we abuse notation and omit the pullback by a section.

LemMA. $\psi=(\sqrt{-1} / 2) \sum \omega_{\alpha \bar{\mu}} \wedge \bar{\omega}_{\alpha \bar{\mu}}$ is a Kähler form on $G(p, n)$; i.e., $d \psi=0$.

Proof. Fix $x_{0} \in G(p, n)$; it certainly suffices to show $d \psi\left(x_{0}\right)=0$. Choose a frame field $s$ according to Corollary (1.19) so that $s^{*}\left(\omega_{\alpha \bar{\beta}}\right)\left(x_{0}\right)=s^{*}\left(\omega_{\mu \bar{\nu}}\right)\left(x_{0}\right)=0$. By the structure equations (1.14), $d \omega_{\alpha \bar{\mu}}=\sum \omega_{\alpha \bar{\beta}} \wedge \omega_{\beta \bar{\mu}}+\sum \omega_{\alpha \bar{\nu}} \wedge \omega_{\nu \bar{\mu}}$. Pulling back by $s$ and evaluating at $x_{0}$, we get 0 , so certainly $d \psi\left(x_{0}\right)=0$, since the statement is independent of choice of frame field.

We remark that since $\psi$ is the invariant Kähler form on $G / H \simeq G(p, n)$, the invariant volume form on $G(p, n)$ is unique (up to constants) and is given, according to Lemma $(1.1)$, by $\psi^{d(p, n)} / d(p, n)$ !, where $d(p, n)=p(n-p)=$ $\operatorname{dim}_{\mathbf{C}} G(p, n)$. For future reference, we record this as a

(1.20) LEMMA. The form $d L=(\sqrt{-1} / 2)^{p(n-p)} \Lambda \omega_{\alpha \bar{\mu}} \wedge \bar{\omega}_{\alpha \bar{\mu}}$ is the invariant volume form on $G(p, n)$.

The universal bundles on the Grassmannian will play a pivotal rôle in our considerations, and so we pause to discuss the relevant geometry. On $G(p, n)$ there 
are the universal sub- and quotient-bundles, of rank $p$ and $n-p$ respectively, defined by the exact sequence

$$
0 \rightarrow E \rightarrow \varepsilon^{n} \rightarrow Q \rightarrow 0 .
$$

$E$ is the holomorphic vector bundle whose fiber over a $p$-plane $\Lambda$ is $\left\{z \in \mathbf{C}^{n}\right.$ : $z \in \Lambda$ \}. Note that this is an immediate generalization of the tautological line bundle $H^{-1}$ on $\mathbf{P}^{n}=G(1, n+1)$. The bundles $E$ and $Q$ inherit natural hermitian structures from the metric on $\mathbf{C}^{n}$.

(1.21) Proposition. Let $e_{1}, \ldots, e_{p}$ be a local unitary frame field for $E$; let $s$ be any local section of $G \rightarrow G / H$ whose first $p$ components are $e_{1}, \ldots, e_{p}$, and write $\omega_{i \bar{j}}=s^{*}\left(\omega_{i j}\right)$ as forms locally defined on $G(p, n)$. Then $\left(\omega_{\alpha \bar{\beta}}\right)$ are the canonical connection forms for $E$ and $\Omega_{\alpha \bar{\beta}}=-\sum \omega_{\alpha \bar{\mu}} \wedge \bar{\omega}_{\beta \bar{\mu}}$ are the curvature forms.

Proof. Immediate.

The interplay between the differential geometry of the Grassmannians and algebro-geometric questions comes from the Schubert cycles whose definition we now recall.

(1.22) Definition. Let $\{0\}=V_{0} \subset V_{1} \subset \cdots \subset V_{n}=\mathbf{C}^{n}$ be a complete flag for $\mathbf{C}^{n}$. Let $a=\left(a_{1}, \ldots, a_{p}\right)$ be a nonincreasing sequence of integers between 0 and $n-p$. Then we define the Schubert cycle

$$
\sigma_{a}=\sigma_{a_{1} \ldots a_{p}}=\left\{\Lambda \in G(p, n): \operatorname{dim}\left(\Lambda \cap V_{n-p+i-a_{i}}\right)>i, i=1, \ldots, p\right\} .
$$

The salient result is the following

(1.23) ThEOREM. $\sigma_{a}$ is an algebraic subvariety of codimension $|a|=\sum a_{i}$. The Poincare duals of the homology classes carried by the basic Schubert cycles $\sigma_{1} \sim_{q}$ and $\sigma_{q}$ are $(-1)^{q} c_{q}(E)$ and $c_{q}(Q)$ respectively.

Proof. [14, pp. 410-411], [27].

(d) Invariant cohomology. Let $G$ be a compact Lie group which acts on a manifold $X$ on the right. We say a differential form $\phi$ on $X$ is invariant if $R_{g}^{*} \phi=\phi$ for all $g \in G$. Let $\operatorname{Inv}^{k}(X)$ denote the invariant complex-valued $k$-forms on $X$; then the invariant forms form a complex $\operatorname{Inv}^{*}(X)$ with respect to the exterior derivative d. $\tilde{\phi}$.

(1.24) Lemma. Any closed form $\phi$ on $X$ is cohomologous to a closed invariant form

Proof. Cf. [25, p. 455].

(1.25) TheOREM. If a compact group $G$ acts on $X$ and $\operatorname{Inv}^{*}(X)$ is the complex of invariant forms, then

$$
H^{k}(X, \mathbf{C}) \cong H^{k}\left(\operatorname{Inv} v^{*}(X)\right)=\frac{\{\text { closed invariant } k \text {-forms }\}}{d\left(\operatorname{Inv} v^{k-1}(X)\right)}
$$

Proof. There is a natural mapping $H^{k}\left(\operatorname{Inv}^{*}(X)\right) \rightarrow H_{D R}^{k}(X) \cong H^{k}(X, \mathrm{C})$. That this map is surjective follows from Lemma (1.24). On the other hand, the mapping is injective, for if an invariant form $\phi=d \rho$ is exact, we can write $\phi=\tilde{\phi}=d \tilde{\rho}=$ $\widetilde{d \rho}$, and thus $[\phi]=0 \in H^{k}\left(\operatorname{Inv}^{*}(X)\right)$. 
In particular, a more restrictive geometric structure leads to a stronger result.

(1.26) COROllaRY. If $X$ is a hermitian (or Riemannian) symmetric space, then any invariant form is closed. As a consequence, $H^{*}(X, \mathrm{C})=\operatorname{Inv}^{*}(X)$.

Proof. By definition [17, p. 225], if $X=G / H$ is a symmetric space, then there is an involution $\sigma$ of $G$ which induces the map $v \leadsto-v$ on each tangent space of $X$. Let $\left\{X_{\mu}\right\}=\left\{X_{p+1}, \ldots, X_{n}\right\}$ be a basis for $\mathfrak{h}$ and add $\left\{X_{\alpha}\right\}=\left\{X_{1}, \ldots, X_{p}\right\}$ to obtain a basis for $\mathrm{g}$. Then $\sigma_{*} X_{\alpha}=-X_{\alpha}$ and $\sigma_{*} X_{\mu}=X_{\mu}$. The structure equations of the Lie group are $\left[X_{i}, X_{j}\right]=\sum c_{i j}^{k} X_{k}$. Since $\sigma_{*}$ is a Lie algebra automorphism, $\sigma_{*}\left[X_{i}, X_{j}\right]=\left[\sigma_{*} X_{i}, \sigma_{*} X_{j}\right]$. In particular, we have, on one hand, $\sigma_{*}\left[X_{\alpha}, X_{\beta}\right]=$ $\left[X_{\alpha}, X_{\beta}\right]=\Sigma c_{\alpha \beta}^{i} X_{i}$, and on the other, $\sigma_{*}\left[X_{\alpha}, X_{\beta}\right]=\sigma_{*}\left(\sum c_{\alpha \beta}^{i} X_{i}\right)=-\Sigma c_{\alpha \beta}^{\gamma} X_{\gamma}+$ $\sum c_{\alpha \beta}^{\mu} X_{\mu}$. Juxtaposing the two, we see that $c_{\alpha \beta}^{\gamma}=0$.

Dualizing, we let $\omega^{1}, \ldots, \omega^{p}, \omega^{p+1}, \ldots, \omega^{n}$ be the Maurer-Cartan forms of $G$; they satisfy $d \omega^{k}=-\frac{1}{2} \sum c_{i j}^{k} \omega^{i} \wedge \omega^{j}$, and therefore we have

$$
d \omega^{\alpha}=-\sum c_{\beta \mu}^{\alpha} \omega^{\beta} \wedge \omega^{\mu}-\frac{1}{2} \sum c_{\mu \nu}^{\alpha} \omega^{\mu} \wedge \omega^{\nu} \equiv 0 \bmod \left(\omega^{\mu}\right) .
$$

Any invariant form $\phi$ on $G / H$ must be a polynomial in $\omega^{\alpha}$ with constant coefficients (since $G$ acts transitively on $G / H$ ), and thus $d \phi \equiv 0 \bmod \left(\omega^{\mu}\right)$. Since $\phi$, and hence $d \phi$, is well defined on $G / H$ we must therefore have $d \phi=0$.

The latter statement follows immediately from Theorem (1.25).

The main point here is that the Grassmannians are symmetric spaces (let

$$
\left.\sigma=\operatorname{Ad}\left[\begin{array}{c|c}
-I & 0 \\
\hline 0 & I
\end{array}\right]\right),
$$

while the more general flag manifolds with which we shall be concerned are not. For further discussion of the cohomology of symmetric spaces vis-à-vis invariant theory, we refer the reader to [1].

By virtue of Corollary (1.26) the question of understanding the cohomology of a homogeneous space is reduced to a computation of invariant forms. There is a subtle switch which occurs, however. We know that all the invariant forms on $G$ are polynomials with constant coefficients in the Maurer-Cartan forms. According to (1.15), certain of these Maurer-Cartan forms give, locally, a basis for forms on $G / H$. Thus, finding invariant forms on $G / H$ is rather a matter of finding those polynomials in the Maurer-Cartan forms which are invariant under the left (or adjoint) action of $H$. Furthemore, since $G$ acts transitively on $G / H$, we are reduced to considering invariant theory on a particular vector space-namely, the tangent space to $G / H$ at a fixed point. This, then, is the great virtue of the homogeneous spaces.

The main tool in these proofs is a slight modification of the most elementary of Hermann Weyl's results on invariant theory.

(1.27) TheOREM (H. WeYL [30]). Let $V$ be an $N$-dimensional hermitian vector space on which $U(N)$ acts. Let $v_{1}, \ldots, v_{k} \in V$. Any $U(N)$-invariant polynomial in the components of the vectors $v_{i}$ and their conjugates is a polynomial in the inner products $\left(v_{i}, v_{j}\right), 1 \leqslant i, j \leqslant k$. 
This theorem was the foundation of Chern's original definition of Chern classes [3] and also of his work on the kinematic formula in Riemannian geometry [6].

Earlier, we observed that $\Sigma \omega_{\alpha \bar{\mu}} \wedge \bar{\omega}_{\alpha \bar{\mu}}$ is an example of an expression in the Maurer-Cartan forms of $U(n)$ which descends to a well-defined form on $G(p, n)$. From a similar computation one might deduce the invariance of any expression of the form

$$
\sum \omega_{\alpha_{1} \bar{\mu}_{1}} \wedge \cdots \wedge \omega_{\alpha_{k} \bar{\mu}_{k}} \wedge \bar{\omega}_{\alpha_{1} \bar{\mu}_{(1)}} \wedge \cdots \wedge \bar{\omega}_{\alpha_{k} \bar{\mu}_{(k)}}
$$

(sum over all $\alpha_{1}, \ldots, \alpha_{k}, \mu_{1}, \ldots, \mu_{k}, \sigma$ an arbitrary permutation), which conforms to the summation convention (each index appearing with a conjugate companion, and summed). With the aid of Weyl's theorem one may now prove a stronger result classifying all invariant forms on the Grassmannian.

(1.28) THEOREM (CHERN [3]). The invariant forms on $G(p, n)$ are polynomials with constant coefficients in the Chern forms $c_{i}\left(\Omega_{E}\right)$ of the universal subbundle (or, alternatively, in the Chern forms $c_{i}\left(\Omega_{Q}\right)$ of the quotient bundle). Hence the Chern classes $c_{i}(E)$ generate $H^{*}(G(p, n), \mathbf{R})$.

We defer the proof to the Appendix since the result is well known. We include the proof since we shall need the same methods later. In particular, we have the

(1.29) Corollary. The invariant forms on $\mathbf{P}^{n}$ are polynomials with constant coefficients in the Kähler form w; i.e., the cohomology is generated by the Chern class of the hyperplane bundle $H$.

2. Crofton formulas in complex integral geometry. The general setting in integral geometry involves two spaces (e.g., manifolds or analytic varieties) $X$ and $Y$ upon which a group acts and some subset $I \subset X \times Y$ which is invariant under the induced action of the group on the product. In the cases which interest us in the complex realm, $X$ and $Y$ will be compact complex manifolds (perhaps with boundary) and the (not necessarily nonsingular) subvariety $I$ will be some sort of incidence correspondence. Moreover, the projection maps

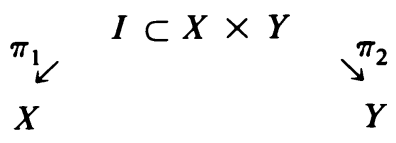

will fiber $I$ over each of $X$ and $Y$. We will concentrate on studying the mapping $\pi_{1 *} \pi_{2}^{*}$ carrying differential forms on $Y$ to those on $X$ (or, when we can afford the sloppiness, the induced mapping of cohomology classes).

When the fiber of $\pi_{2}$ is zero-dimensional, the situation becomes particularly simple. We shall refer to formulas which arise in such a manner as Crofton formulas $^{1}$ for they are formally analogous to the formulas originally considered by Crofton (cf. [23], [2]).

\footnotetext{
${ }^{1}$ Note that this is not in keeping with Griffiths' nomenclature in [12]. He seems to refer to any integro-geometric formula as a Crofton formula. We shall try to be more specific and shall refer to more general sorts of formulas as kinematic formulas, as in [4], [6].
} 
We start with formulas of the Crofton type because here both the topological and the invariant-theoretic considerations appear in the simplest and clearest light. The first formula is the most elementary insofar as volume is the only geometric invariant to enter; in the two Gauss-Bonnet formulas which follow, however, the geometry of the tangent bundle begins to appear.

(2.1) Theorem (Crofton formula for a SUbVARIETy of $\mathbf{P}^{n}$ ). Let $X^{k} \subset \mathbf{P}^{n}$ be $a$ compact analytic subvariety with boundary (perhaps empty). Let $d L$ denote the invariant measure on $\mathbf{G}(n-k, n)^{2}$ (cf. Lemma (1.20)), and for $L \in \mathbf{G}(n-k, n)$, let $n(X \cap L)=$ the number of points of intersection of $X$ and $L$. Then

$$
\int_{\mathbf{G}(n-k, n)} n(X \cap L) d L=\operatorname{vol}(\mathbf{G}(n-k, n)) \int_{X} \omega^{k}=\frac{\operatorname{vol}(\mathbf{G}(n-k, n))}{\operatorname{vol}\left(\mathbf{P}^{k}\right)} \operatorname{vol}(X),
$$

where $\omega=(\sqrt{-1} / 2 \pi) \partial \bar{\partial} \log \|z\|^{2}$ is the normalized Kähler form on $\mathbf{P}^{n}$.

Proof. The main point is to prove a universal, yet local, formula on $\mathbf{P}^{n}$ and introduce the particular subvariety $X$ only at the last moment. Consider the incidence correspondence $I=\{(z, L): z \in L\} \subset \mathbf{P}^{n} \times \mathbf{G}(n-k, n)$, together with projections:

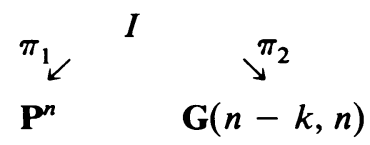

Here the group $U(n+1)$ acts on the entire picture. Note, however, that when we restrict to $X^{k} \subset \mathbf{P}^{n}$, the fiber $\pi_{2}^{-1}(L)$ will consist of the points of $X$ lying in $L \in \mathbf{G}(n-k, n)$ and by transversality this is generically a finite set. We hope this helps explain our earlier comments on terminology.

Computing $\pi_{1 *}\left(\pi_{2}^{*} d L\right)$ is the crucial step; this will be a form of real degree $=$ $\operatorname{dim} \mathbf{G}(n-k, n)-\operatorname{dim}$ fiber $\pi_{1}=\operatorname{dim} \mathbf{P}^{n}-\operatorname{dim}$ fiber $\pi_{2}=2(n-(n-k))=2 k$ (all dimensions real). On the other hand, it is certainly invariant under $U(n+1)$ and of type $(k, k)$; it is therefore a multiple of $\omega^{k}$ by Corollary (1.29).

We can, however, give a more directly topological argument using Poincaré duality (cf. Appendix (a)). For any closed form $\Phi$ on $\mathbf{G}(n-k, n)$, the Poincaré dual of $\pi_{1 *} \pi_{2}^{*} \Phi$ is $\pi_{1 *}\left(\pi_{2}^{-1} Z\right)$, where $Z=\mathscr{D}^{-1}(\Phi)$ is the homology class representing the dual of $\Phi$. In this case, if we normalize the volume of $\mathbf{G}(n-k, n)$ to be one, $Z$ is a point $L_{0} \in \mathbf{G}(n-k, n)$ and clearly $\pi_{1 *}\left(\pi_{2}^{-1} Z\right) \cong\left\{z \in L_{0} \subset \mathbf{P}^{n}\right\}$, whose Poincaré dual is precisely $\omega^{k}$. We hasten to emphasize that despite appearances to the contrary, we have not avoided invariant theory by this route. This computation shows merely that the cohomology class of $\pi_{1 *}\left(\pi_{2}^{*} d L\right)$ is $\left[\omega^{k}\right] \in H^{2 k}\left(\mathbf{P}^{n}, \mathrm{C}\right)$. However, since the subvariety $X$ may have boundary, it is not sufficient to know just the cohomology class-we need the precise form representing the class. Now, by Corollary (1.26) there is a unique invariant form representing the class, and since $\omega^{k}$ is invariant, we are done.

To finish the proof, we consider the inclusion $X \hookrightarrow \mathbf{P}^{n}$ and chase back the diagram (see Figure 3).

\footnotetext{
${ }^{2}$ Recall that $\mathbf{G}(k, n) \simeq G(k+1, n+1)$ is the space of $\mathbf{P}^{k}$ 's in $\mathbf{P}^{n}$.
} 


$$
\begin{aligned}
\int_{X} \pi_{1 *} & \left(\pi_{2}^{*} d L\right)=\int_{\pi_{1}^{-1}(X)} \pi_{2}^{*} d L=\int_{\mathbf{G}(n-k, n)} n(X \cap L) d L \\
& \| \int_{X} \omega^{k}
\end{aligned}
$$

It remains to evaluate the constant $c$. This is easily done by taking $X=\mathbf{P}^{k} \hookrightarrow \mathbf{P}^{n}$, whence the result follows. Note, by the way, that there is no trouble with singularities of $X$ since they occur in real codimension at least two (cf. [12], [14]).

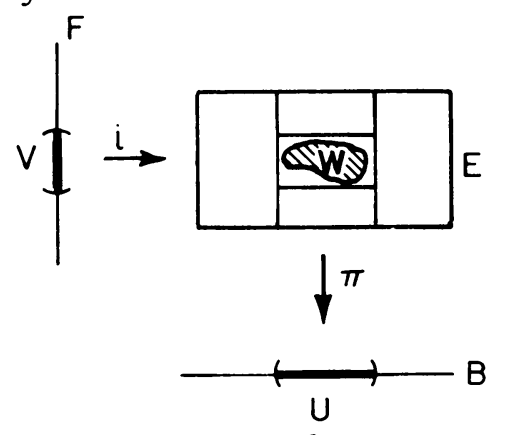

FIGURE 3

REMARK. This Crofton formula may be viewed as an integrated form of Bezout's Theorem: if $X$ is compact (hence algebraic), the generic intersection $X \cap L$ will consist of $d=\operatorname{deg} X$ points, and the theorem merely expresses the proportionality of volume and degree. However, the integrated form has proved useful in the noncompact case, particularly in value distribution theory (cf. [11], [13]).

As examples of a more subtle Crofton formula, we consider the extrinsic Gauss-Bonnet theorems in the complex setting. Now the Gauss mapping of a submanifold of $\mathbf{C}^{n}$ or $\mathbf{P}^{n}$ comes naturally into play.

(2.2) Theorem (Complex Gauss-Bonnet IN $\mathbf{C}^{\boldsymbol{n}}$ ). Let $X^{k} \subset \mathbf{C}^{n}$ be a compact complex submanifold with boundary, and let $\Omega_{X}$ be the curvature form (1.7) of the induced hermitian metric on $X$. Let $H \in \mathbf{P}^{n-1 *}$ be a hyperplane through the origin in $\mathrm{C}^{n}$, and let $n(X, H)=$ the number of points $z \in X$ such that $T_{z} X \subset H$ (viewing $T_{z} X$ as a linear subspace of $\left.\mathbf{C}^{n}\right)$. Then

$$
\int_{\mathbf{P}^{n-1 *}} n(X, H) d H=(-1)^{k} \int_{X} c_{k}\left(\Omega_{X}\right) \text { where } \int_{\mathbf{p}^{n-1 *}} d H=1 .
$$

REMARK. (1) There is a comparable integro-geometric formulation of the GaussBonnet theorem for real submanifolds of $\mathbf{R}^{n}$. We shall not go into this here, but we do point out that, as in the real case, we have a juxtaposition of the Euler class and Morse theory. (Cf. e.g. [19].)

(2) This Gauss-Bonnet theorem gives a more direct proof of Langevin's main result in [18] relating curvature and algebraic singularities.

Proof. We first seek an appropriate universal formula on the Grassmannian $G(k, n)$. Let $I=\{(L, H): L \subset H\} \subset G(k, n) \times \mathbf{P}^{n-1 *}$, and define projections:

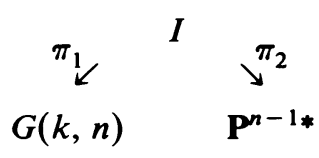


Note that the affine unitary group acts, and that here $I$ is a nonsingular subvariety of $G(k, n) \times \mathbf{P}^{n-1 *}$; in fact, it is the flag manifold $G(k, n-1, n)$. Let $d H$ be the normalized volume element on $\mathbf{P}^{n-1 *}$ and consider $\psi=\pi_{1 *}\left(\pi_{2}^{*} d H\right)$. Note that the fiber of $\pi_{1} \cong\left\{H \supset L_{0}\right\} \cong \mathbf{P}^{n-k-1 *}$, and therefore $\psi$ will be an invariant form of type $(k, k)$ on $G(k, n)$. The precise determination of $\psi$ is slightly more involved here; the direct approach is to chase through Poincaré duality, and to use Definition (1.22) and Theorem (1.23).

$$
\mathscr{D}^{-1}(d H)=\left\{H_{0}\right\} \in \mathbf{P}^{n-1 *} ; \quad \pi_{1 *}\left(\pi_{2}^{-1} H_{0}\right) \cong\left\{L \in G(k, n): L \subset H_{0}\right\}=\sigma_{1} \underbrace{\ldots 1}_{k},
$$

and

$$
\mathscr{D}\left(\sigma_{\underbrace{\ldots 1}_{k}}\right)=(-1)^{k} c_{k}(E),
$$

where $E$ is the universal subbundle on $G(k, n)$. As before, this computation gives just a cohomology class, but this form is in fact the unique invariant form representing the class.

The next step is to specialize to $X \hookrightarrow \mathbf{C}^{n}$. We define the Gauss mapping $\gamma$ : $X \rightarrow G(k, n)$ by $\gamma(z)=T_{z} X$. Then certainly $\gamma^{*} E=T X$, and the theorem follows from the naturality (1.10) of Chern classes:

$$
(-1)^{k} \int_{X} c_{k}\left(\Omega_{X}\right)=\int_{X} \gamma^{*}\left(\pi_{1 *} \pi_{2}^{*} d H\right)=\int_{\pi_{1}^{-1}(\gamma(X))} \pi_{2}^{*} d H=\int_{\mathbf{p}^{n-1 *}} n(X, H) d H,
$$

where the last equality follows from the definition of the incidence correspondence.

Some classical algebraic geometry comes into the picture when we pass to the projective analogue of Theorem (2.2). The appropriate geometric construction is the projective Gauss mapping. Recalling from $\$ 1$ the notion of the projective tangent bundle $\widetilde{T X}$ of $X^{k} \subset \mathbf{P}^{n}$, we define the projective Gauss mapping $\gamma$ : $X \rightarrow \mathbf{G}(k, n)$ by $\gamma(z)=\widetilde{T_{z} X}$. Note that by virtue of this definition, $\gamma^{*} E=\tilde{E}$, where $E$ is the universal subbundle on $\mathbf{G}(k, n)=G(k+1, n+1)$ and $\tilde{E}$ is the vector bundle associated to $\overparen{T X}$. The appropriate theorem is now

(2.3) Theorem (Projective Gauss-Bonnet). Let $X^{k} \subset \mathbf{P}^{n}$ be a compact complex submanifold with boundary (perhaps empty). Let $\tilde{\Omega}_{X}$ denote the curvature form of the hermitian vector bundle $\tilde{E}$ on $X$. For $\xi \in \mathbf{G}(n-2, n)$, let $n(X, \xi)$ denote the number of points $z \in X$ such that $\widetilde{T_{z} X} \cap \xi$ has dimension $\geqslant k-1$. Then

$$
\int_{\mathbf{G}(n-2, n)} n(X, \xi) d \xi=(-1)^{k} \int_{X} c_{k}\left(\tilde{\Omega}_{X}\right) \text { where } \int_{\mathbf{G}(n-2, n)} d \xi=1
$$

Proof. That we are seeking $c_{k}$ of a bundle of rank $k+1$ accounts for the difference between Theorems (2.2) and (2.3). In particular, one checks from the definition (1.22) of Schubert cycles that

$$
\sigma_{\underbrace{}_{\dot{k}} \dot{1}^{1}}(\xi)=\{L \in \mathbf{G}(k, n): \operatorname{dim}(L \cap \xi)>k-1\}
$$

-translating everything into projective notation. The proof is in all other respects identical to that of Theorem (2.2). 


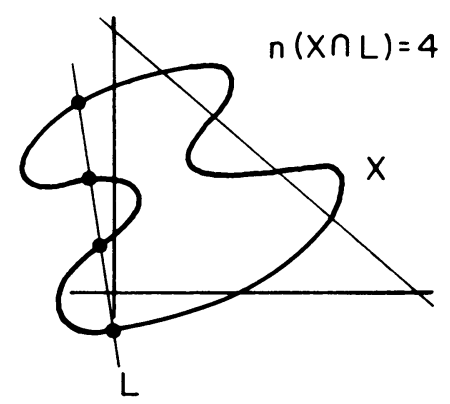

FIGURE 4

We consider a simple, but instructive example. Let $C$ be a nonsingular algebraic curve of degree $d$ in $\mathbf{P}^{2}$. For generic $P \in \mathbf{P}^{2}, n(C, P)$ is a classical invariant called the class of $C$ (see Figure 4). This is the degree $\delta$ of the dual curve $C^{*} \subset \mathbf{P}^{2 *}$. Since $C$ is nonsingular, it is not hard to compute that (cf. $[14$, p. 280])

$$
\delta=d(d-1), \quad g=\text { genus } C=(d-1)(d-2) / 2 .
$$

Now, $-\int_{C} c_{1}\left(\Omega_{C}\right)=-\chi(C)=2 g-2=d(d-3)$, and this is not equal to $\delta=$ $d(d-1)$. The discrepancy, of course, is due to the twist relating $T C$ and $\widetilde{T C}$. In particular, the Euler sequence (1.11) gives

$$
0 \rightarrow H^{-1} \rightarrow \tilde{E} \rightarrow T C \otimes H^{-1} \rightarrow 0,
$$

and thus $c_{1}\left(\tilde{\Omega}_{C}\right)=c_{1}\left(\Omega_{C}\right)-2 \omega$ and $\int_{C} \omega=d$, so $-\int_{C} c_{1}\left(\tilde{\Omega}_{C}\right)=d(d-3)+2 d=$ $d(d-1)$.

Using the formulas (1.13) relating Chern forms of $T X$ to those of $\tilde{E}$, we can rewrite Theorem (2.3) in a somewhat more intrinsic manner; now all intermediate Chern forms appear.

COROLlaRY. $\int_{\mathbf{G}(n-2, n)} n(X, \xi) d \xi=(-1)^{k} \sum_{j=0}^{k}(-1)^{j}(j+1) \int_{X} c_{k-j}\left(\Omega_{X}\right) \wedge \omega^{j}$.

This gives an immediate application generalizing our prior example. If $X^{n-1} \subset$ $\mathbf{P}^{n}$ is a nonsingular algebraic hypersurface, we can define the dual hypersurface $X^{*} \subset \mathbf{P}^{n *}\left(X^{*}=\left\{\widetilde{T_{z} X}: z \in X\right\}\right)$. The degree $\delta$ of $X^{*}$ is precisely $n(X, \xi)$ for generic $\xi$, by projective duality. We therefore have the formula

$$
\delta=(-1)^{n-1} \sum_{j=0}^{n-1}(-1)^{j}(j+1) \int_{X} c_{n-j-1}\left(\Omega_{X}\right) \wedge \omega^{j}
$$

REMARK. We point out that there are generalizations of Theorems (2.2) and (2.3) to arbitrary Schubert cycles in the Grassmannian. One must take greater care with the proof, however, since the general Schubert cycle is singular and therefore the incidence correspondence becomes a singular variety. Integration over the fiber is nevertheless valid. Griffiths gives some discussion of these formulas in [12].

3. The linear kinematic formula in $\mathbf{P}^{n}$. We now move on to discuss integro-geometric formulas which are not of the Crofton type. The question is this: to what extent can we recover geometric information about a complex submanifold of $\mathbf{P}^{n}$ 
from that of its plane sections? In the algebraic case, there is an immediate answer, and it is with this case that we begin. In the nonalgebraic case, however, we must resort to an averaging process to eradicate the nonintrinsic terms coming from the second fundamental form of the submanifold's sections.

Proposition. Let $X^{p} \subset \mathbf{P}^{n}$ be a p-dimensional nonsingular algebraic variety, and let $L \in \mathbf{G}(n-k, n)$ be a linear space of codimension $k$ meeting $X$ transversely. Then we have the following equation of differential forms (and hence of cohomology classes) on $X \cap L$ :

$$
c_{j}\left(\Omega_{X \cap L}\right)=\left.\sum_{i=0}^{j}(-1)^{i}\left(\begin{array}{c}
k+i-1 \\
i
\end{array}\right) \omega^{i} \wedge c_{j-i}\left(\Omega_{X}\right)\right|_{X \cap L}, \quad j=0, \ldots, p-k .
$$

Proof. Since $X$ and $L$ meet transversely, the normal bundle $N(X \cap L, X)$ of $X \cap L$ in $X$ is the restriction to $X \cap L$ of

$$
N\left(L, \mathbf{P}^{n}\right)=\frac{H \oplus \cdots \oplus H}{k \text { times }}=H^{\oplus k} .
$$

From the exact sequence

$$
\left.0 \rightarrow T(X \cap L) \rightarrow T X\right|_{X \cap L} \rightarrow N(X \cap L, X) \rightarrow 0
$$

of vector bundles on $X \cap L$, it follows from (1.10) that $c(X)=c(X \cap L)$. $c\left(H^{\oplus k}\right)$, whence $c(X \cap L)=c(X) /(1+\omega)^{k}$. The proposition now follows from an elementary

(3.2) LemMA. As a formal power series, $(1+x)^{-k}=\Sigma(-1)^{i}\left({ }_{i}^{k+i-1}\right) x^{i}$.

We now exploit Poincaré duality to give this in a more convenient form. Since the Poincaré dual of $L \cong \mathbf{P}^{n-k}$ is $\omega^{k}$, we have by (A.4)

$$
\int_{X \cap L} \psi=\int_{X} \psi \wedge \omega^{k} \text { for any closed }(p-k, p-k) \text { form } \psi
$$

In particular, applying (3.1) and (3.3) in turn,

$$
\begin{aligned}
\int_{X \cap L} c_{j}\left(\Omega_{X \cap L}\right) \wedge \omega^{p-k-j} & =\sum(-1)^{i}\left(\begin{array}{c}
k+i-1 \\
i
\end{array}\right) \int_{X \cap L} c_{j-i}\left(\Omega_{X}\right) \wedge \omega^{p-k-j+i} \\
& =\sum(-1)^{i}\left(\begin{array}{c}
k+i-1 \\
i
\end{array}\right) \int_{X} c_{j-i}\left(\Omega_{X}\right) \wedge \omega^{p-j+i}
\end{aligned}
$$

i.e.,

$$
\int_{X \cap L} c_{j}\left(\Omega_{X \cap L}\right) \wedge \omega^{p-k-j}=\sum_{i=0}^{j}(-1)^{i}\left(\begin{array}{c}
k+j-i-1 \\
j-i
\end{array}\right) \int_{X} c_{i}\left(\Omega_{X}\right) \wedge \omega^{p-i} .
$$

We let $d L$ denote the invariant measure on $\mathbf{G}(n-k, n)$ defined in Lemma (1.20). Since the set of $L \in \mathbf{G}(n-k, n)$ which fail to meet $X$ transversely is of measure 
zero (Bertini's Theorem), we may integrate (3.4) over $\mathbf{G}(n-k, n)$ to obtain

$$
\begin{gathered}
\int_{\mathbf{G}(n-k, n)}\left(\int_{X \cap L} c_{j}\left(\Omega_{X \cap L}\right) \wedge \omega^{p-k-j}\right) d L=\sum_{i=0}^{j} a_{i} \int_{X} c_{i}\left(\Omega_{X}\right) \wedge \omega^{p-i}, \\
a_{i}=\operatorname{vol}(\mathbf{G}(n-k, n)) \cdot(-1)^{j-i}\left(\begin{array}{c}
k+j-i-1 \\
j-i
\end{array}\right) .
\end{gathered}
$$

As it stands, this is merely a trivial deduction from (3.4). It is, however, the integrated version (3.5) of (3.1) which holds locally. It is not altogether surprising that one must average over a group (or all linear spaces) in order to get a local result; for if we put $j=0, k=p$, we recover the Crofton formula (2.1) and this is certainly not valid unaveraged for nonalgebraic subvarieties. So we turn to proving the kinematic formula (3.5) for an arbitrary compact submanifold $X$ with boundary.

The key to the proof of the Gauss-Bonnet formulas was a universal formula on a Grassmannian. Here, however, we must introduce a slightly more intricate model space. We define the so-called pointed Grassmannian

$$
\mathbf{G}(0, p, n)=\{(z, T): z \in T\} \subset \mathbf{P}^{n} \times \mathbf{G}(p, n) ;
$$

$\mathbf{G}(0, p, n)$ is a homogeneous space with group $U(n+1)$ but is not symmetric. (This distinction will prove important in comparing cohomology and invariant forms.) On $\mathbf{G}(0, p, n)$ there is the tautological bundle $E_{p+1}$ of rank $p+1$ obtained by pullback from $\mathbf{G}(p, n)$, as well as the pullback $H^{-1}$ of the tautological line bundle on $\mathbf{P}^{n}$. Note that $H^{-1}$ is naturally a subbundle of $E_{p+1}$.

Since we want to relate $X \cap L$ to $X$, fixing $z_{0} \in X$ amounts to fixing a $p$-plane $\widetilde{T_{z_{0}} X}=T \in \mathbf{G}(p, n)$ and examining all $(p-k)$-planes $S$ obtained by intersecting $T$ with a variable $(n-k)$-plane $L \in \mathbf{G}(n-k, n)$. So we are led to a more elaborate flag space. Define

$$
\begin{aligned}
\mathbf{G}(0, p-k, n & -k, n) \\
& =\{(z, S, L): z \in S \subset L\} \subset \mathbf{P}^{n} \times \mathbf{G}(p-k, n) \times \mathbf{G}(n-k, n) .
\end{aligned}
$$

The marvelous observation is that the structure of this flag space embodies the geometry we wish to study in a universal way. What makes everything work is the linear structure of the projective tangent bundle; that is, if $L$ meets $X$ transversely, then $\overparen{T_{z}(X \cap L)}=\widetilde{T_{z} X} \cap L$. Translating what we have just said into an incidence correspondence, we define

$$
\begin{gathered}
I \subset \mathbf{G}(0, p, n) \times \mathbf{G}(0, p-k, n-k, n), \\
I=\left\{(z, T),\left(z^{\prime}, S, L\right): z=z^{\prime}, S \subset T \cap L\right\} \\
\pi_{1} \\
\begin{array}{l}
\mathbf{G}(0, p, n) \\
\pi_{2}
\end{array} \\
\mathbf{G}(0, p-k, n-k, n)
\end{gathered}
$$

and observe that $U(n+1)$ acts on all three spaces on the right. We with to understand the mapping $\pi_{1 *} \pi_{2}^{*}$.

Now, the computation of all invariant forms on $\mathbf{G}(0, p, n)$ is complicated by the fact that $\mathbf{G}(0, p, n)$ is homogeneous with group $U(n+1)$, but is not a symmetric 
space. For our immediate purposes, only the closed forms-i.e., those with topological meaning-will be important, and so to simplify our considerations we discard the others. It is a quirk of fate that the geometry of the Gauss mapping embodied in the differential ideal 9 which follows does this automatically. To drive home the point that there are forms on a nonsymmetric homogeneous space which are not closed, we give two simple examples. Define $\phi=\Sigma \Omega_{\alpha \bar{\beta}} \wedge \omega^{\alpha} \wedge \omega^{\bar{\beta}}, \psi=\Sigma \omega_{\alpha \bar{\mu}} \wedge$ $\omega^{\alpha} \wedge \omega^{\bar{\mu}}$. Using a local frame field in which $\omega_{i \bar{j}}=\omega_{\mu \bar{\nu}}=0$ at $\left(z_{0}, T_{0}\right)$ and applying the structure equations (3.1), we find that

$$
d \phi=\sum \Omega_{\alpha \bar{\beta}} \wedge\left(\omega^{\mu} \wedge \omega_{\mu \bar{\alpha}} \wedge \omega^{\bar{\beta}}-\omega^{\alpha} \wedge \omega^{\bar{\mu}} \wedge \bar{\omega}_{\mu \bar{\beta}}\right)
$$

and

$$
d \psi=-\left(\sum \Omega_{\nu \bar{\mu}} \wedge \omega^{\nu} \wedge \omega^{\bar{\mu}}+\sum \Omega_{\alpha \bar{\beta}} \wedge \omega^{\alpha} \wedge \omega^{\bar{\beta}}\right) .
$$

Note, in particular, that $\psi$ is a form of odd degree, and by Theorem (1.25) there can be no nonzero closed form of odd degree (cf. Appendix). The crucial observation, however, is that if we compute modulo the differential ideal $\mathscr{G}=\left(\omega^{\mu}, \omega^{\bar{\mu}}\right)$, then these examples disappear. In particular, $\psi \equiv 0$, and $\phi=\Sigma \omega_{\alpha \bar{\mu}} \wedge \omega_{\mu \bar{\beta}} \wedge \omega^{\alpha} \wedge \omega^{\bar{\beta}}=$ $\sum \omega^{\alpha} \wedge \omega_{\alpha \bar{\mu}} \wedge \omega_{\mu \bar{\beta}} \wedge \omega^{\bar{\beta}}=\Sigma d \omega^{\mu} \wedge \omega_{\mu \bar{\beta}} \wedge \omega^{\bar{\beta}} \equiv 0$.

We now modify the notion of adapted unitary frames defined on Grassmannians in $\$ 1(c)$.

We use the following index ranges:

$$
1 \leqslant A, B, C \leqslant n, \quad 1 \leqslant \alpha, \beta, \gamma \leqslant p, \quad p+1 \leqslant \mu, \nu \leqslant n, \quad 0<i, j<p .
$$

We say $e_{0}, \ldots, e_{n}$ is an adapted frame field on a subset of $\mathbf{G}(0, p, n)$ if, at $(z, T), e_{0}$ projects to $z \in \mathbf{P}^{n}$ and $e_{0}, \ldots, e_{p}$ are a frame for $T$. Now, since $\mathbf{G}(0, p, n) \simeq$ $U(n+1) /(U(1) \times U(p) \times U(n-p))$, we see that the locally defined forms $\omega^{1}, \ldots, \omega^{n}\left(\omega^{A}=\omega_{0 \bar{A}}\right), \omega_{\alpha \bar{\mu}}$ span the $(1,0)$ cotangent space of $\mathbf{G}(0, p, n)$.

We cite the result from invariant theory which we need at this point. We defer the proof to the Appendix.

(3.7) Theorem. Modulo the differential ideal $G=\left(\omega^{\mu}, \omega^{\bar{\mu}}\right)$, any invariant form on $\mathbf{G}(0, p, n) \cong U(n+1) /(U(1) \times U(p) \times U(n-p))$ is a polynomial with constant coefficients in the (truncated) Kähler form $\omega=\sum \omega^{\alpha} \wedge \omega^{\bar{\alpha}}$ and the Chern forms $c_{k}\left(\Omega_{i j}\right)$ of the universal bundle $E_{p+1}$ on $\mathbf{G}(0, p, n)$.

We are now in a position to prove the

(3.8) TheOREM (LiNeAR KINEMATIC FORMUla). Let $X^{p} \subset \mathbf{P}^{n}$ be a complex submanifold (perhaps with boundary). Then for $j=0, \ldots, p-k$,

$$
\begin{gathered}
\int_{\mathbf{G}(n-k, n)}\left(\int_{X \cap L} c_{j}\left(\Omega_{X \cap L}\right) \wedge \omega^{p-k-j}\right) d L=\sum_{i=0}^{j} a_{i} \int_{X} c_{i}\left(\Omega_{X}\right) \wedge \omega^{p-i}, \\
a_{i}=\operatorname{vol}(\mathbf{G}(n-k, n)) \cdot(-1)^{j-i}\left(\begin{array}{c}
k+j-i-1 \\
j-i
\end{array}\right),
\end{gathered}
$$

whenever both sides make sense. 
Proof. Step 1. We first derive an appropriate universal formula using (3.6). Define $\psi_{j}=c_{j}\left(\Omega_{E_{p-k+1}}\right) \wedge \omega^{p-k-j} \wedge d L$ on $\mathbf{G}(C, p-k, n-k, n)$, where $d L$ is the invariant measure on $\mathbf{G}(n-k, n) . \psi_{j}$ is a form of degree $2[p+(n-k) k]$ on $\mathbf{G}(0, p-k, n-k, n)$ and the fiber of $\pi_{1}$ consists of all $(n-k)$-planes passing through a fixed point and is therefore isomorphic to $\mathbf{G}(n-k, n)$; hence, $\pi_{1 *} \pi_{2}^{*} \psi_{j}$ is an invariant form of total degree $2 p$ on $\mathbf{G}(0, p, n)$.

From (3.7), then, we know that $\pi_{1 *} \pi_{2}^{*} \psi_{j}$ can be written, modulo $q$, as some polynomial with constant coefficients $P_{j}\left(\Omega_{E_{p+1}}, \omega\right)$ of total degree $2 p$ in the Chern forms of $E_{p+1}$ and the Kähler form $\omega$.

Step 2. We next apply these results to deduce a formula for an arbitrary submanifold $X^{p} \subset \mathbf{P}^{n}$. The trick here is to consider a pointed projective Gauss mapping. We now define $\gamma: X \rightarrow \mathbf{G}(0, p, n), \gamma(z)=\left(z, \widetilde{T_{z} X}\right)$. Note that (a) $\gamma$ is automatically an embedding, (b) $\gamma^{*} \omega_{\mathrm{p}^{n}}=\omega_{X}$ and (c) $\gamma^{*} E_{p+1}=\tilde{E}$ (cf. $\S 1($ a)). From this we see that $\gamma^{*} P_{j}\left(\Omega_{E_{p+1}}, \omega\right)=P_{j}\left(\tilde{\Omega}_{X}, \omega\right)$, and from (1.13) we infer that there is a universal polynomial $P_{i}^{\prime}\left(\Omega_{X}, \omega\right)$ of degree $2 p$ such that $P_{j}^{\prime}\left(\Omega_{X}, \omega\right)=P_{j}\left(\Omega_{X}, \omega\right)$. By universal we mean that the coefficients of the polynomial depend on dimensions but not on the particular submanifold $X$.

Now we perform the integration. Since $\gamma$ is an embedding,

$$
\begin{aligned}
\int_{X} P_{j}\left(\tilde{\Omega}_{X}, \omega\right) & =\int_{X} \gamma^{*}\left(\pi_{1 *} \pi_{2}^{*} \psi_{j}\right)=\int_{\gamma(X)} \pi_{1 *} \pi_{2}^{*} \psi_{j} \\
& =\int_{\pi_{1}^{-1}(\gamma(X))} \pi_{2}^{*} \psi_{j}=\int_{\bar{X}} \psi_{j},
\end{aligned}
$$

where $\bar{X}=\pi_{2}\left(\pi_{1}^{-1}(\gamma(X))\right)$. Note that $\left.\pi_{2}\right|_{\pi_{1}^{-1}(\gamma(X))}$ is automatically a biholomorphism since we have used the pointed Gauss mapping. An application of Fubini's theorem gives

$$
\int_{\bar{X}} \psi_{j}=\int_{\substack{L \in \mathbf{G}(n-k, n) \\ L \pitchfork X}}\left(\int_{\bar{X} \cap p^{-1}(L)} c_{j}\left(\Omega_{E_{p-k+1}}\right) \wedge \omega^{p-k-j}\right) d L,
$$

where $p: \mathbf{G}(0, p-k, n-k, n) \rightarrow \mathbf{G}(n-k, n)$ is the obvious projection. Note that we are integrating here only over those $L$ meeting $X$ transversely. The complement, of course, has measure zero, and in $\$ 4$ we will check questions of convergence very carefully in a more general setting.

The crucial observation is that whenever $X 历 L, \widehat{T_{z}(X \cap L)}=\widetilde{T_{z} X} \cap L$ and therefore if we define the Gauss mapping of $X \cap L$

$$
\gamma_{L}: X \cap L \rightarrow \mathbf{G}(0, p-k, n)
$$

we have

$$
\begin{aligned}
\bar{X} \cap p^{-1}(L) & \cong\left\{\left(z, \widetilde{T_{z} X} \cap L\right): z \in X\right\} \subset \mathbf{G}(0, p-k, n) \\
& \cong \gamma_{L}(X \cap L)
\end{aligned}
$$


Thus,

$$
\begin{aligned}
\int_{\bar{X} \cap p^{-1}(L)} c_{j}\left(\Omega_{\left.E_{p-k+1}\right) \wedge \omega^{p-k-j}}\right. & =\int_{X \cap L} \gamma_{L}^{*}\left(c_{j}\left(\Omega_{E_{p-k+1}}\right) \wedge \omega^{p-k-j}\right) \\
& =\int_{X \cap L} c_{j}\left(\gamma_{L}^{*} \Omega_{E_{p-k+1}}\right) \wedge \omega^{p-k-j} .
\end{aligned}
$$

Now, $c_{j}\left(\gamma_{L}^{*} \Omega_{E_{p-k+1}}\right)$ may similarly be expressed in a universal manner as a polynomial in the Kähler form and Chern forms $c_{i}\left(\Omega_{X \cap L}\right), 0<i<j$. By induction, therefore, one may evaluate

$$
\int_{\mathbf{G}(n-k, n)}\left(\int_{X \cap L} c_{j}\left(\Omega_{X \cap L}\right) \wedge \omega^{p-k-j}\right) d L
$$

in terms of universal constants and integrals of the form $\int_{X} c_{i}\left(\Omega_{X}\right) \wedge \omega^{p-i}$.

Step 3. We have only to determine the constants. Since they are independent of $X$, however, formula (3.5) derived earlier gives the result. The proof is now complete.

In examining the proof, we notice that we never explicitly needed the particularly simple form of $\psi_{j}$. Indeed, the same proof and a similar algebraic lemma replacing (3.1) give the following

(3.10) TheORem. Let $P(\Omega, \omega)$ be a polynomial of total degree $2(p-k)$ in Chern form $c_{i}(\Omega)$ and Kähler form $\omega$. Then there is canonically associated to it a polynomial $Q(\Omega, \omega)$ of total degree $2 p$ so that

$$
\int_{\mathbf{G}(n-k, n)}\left(\int_{X \cap L} P\left(\Omega_{X \cap L}, \omega\right)\right) d L=\int_{X} Q\left(\Omega_{X}, \omega\right)
$$

for every submanifold $X^{p} \subset \mathbf{P}^{n}$ for which both sides make sense.

4. The general kinematic formula in $\mathbf{P}^{n}$. We now turn to the extension of the linear kinematic formula (3.8) to nonlinear spaces. This is geometrically much more interesting and should have various applications. Proceeding as in the linear case, we will first derive an algebro-geometric formula and then prove the corresponding local theorem by doing invariant theory in an appropriate universal setting; this time some tricky modifications will be necessary.

To begin, we examine the (compact) algebraic case.

Proposition. Let $X^{p}, Y^{q} \subset \mathbf{P}^{n}$ be nonsingular algebraic varieties intersecting transversely. Then for any $j=0, \ldots, p+q-n$, we have the equation of differential forms (and hence of cohomology classes) on $X \cap Y$ :

$$
c_{j}\left(\Omega_{X \cap Y}\right)=\left.\sum_{\substack{i, l \\
0<i+l<j}}(-1)^{j-i-l}\left(\begin{array}{c}
n+j-i-l \\
n
\end{array}\right) \omega^{j-i-l} \wedge c_{i}\left(\Omega_{X}\right) \wedge c_{l}\left(\Omega_{Y}\right)\right|_{X \cap Y}
$$

Proof. Consider the exact sequence

$$
\left.0 \rightarrow T(X \cap Y) \rightarrow T X\right|_{X \cap Y} \rightarrow N(X \cap Y, X) \rightarrow 0
$$


of vector bundles on $X \cap Y$. Since $X$ and $Y$ intersect transversely, $N(X \cap Y, X)$ $=\left.N\left(Y, \mathbf{P}^{n}\right)\right|_{X \cap Y}$. Therefore we deduce, using (1.10), the equation of total Chern classes on $X \cap Y$,

$$
c(X \cap Y)=\frac{c(X)}{c(N(X \cap Y, X))}=\frac{c(X)}{c\left(N\left(Y, \mathbf{P}^{n}\right)\right)}=\frac{c(X) c(Y)}{c\left(\mathbf{P}^{n}\right)} .
$$

Applying (1.12) and Lemma (3.2), we have $c(X \cap Y)=c(X) c(Y)$. $\Sigma(-1)^{k}\left(\begin{array}{c}n+k \\ n\end{array}\right) \omega^{k}$, and (4.1) follows immediately.

Next we want to give (4.1) in integrated form, using Poincaré duality. For this we shall need the

(4.2) Lemma. Let $X^{p}, Y^{q} \subset \mathbf{P}^{n}$ be compact complex submanifolds meeting transversely. Let $\phi$ and $\psi$ be closed forms of type $(k, k)$ and $(l, l)$ on $X$ and $Y$ respectively. Then

$$
\int_{X \cap Y} \phi \wedge \psi \wedge \omega^{p+q-n-k-l}=\int_{X} \phi \wedge \omega^{p-k} \int_{Y} \psi \wedge \omega^{q-l}, \quad p+q>n+k+l .
$$

Proof. Note that it is an immediate consequence of Poincare duality (A.4) that if $M^{m}$ is a compact, oriented smooth manifold and $N^{n}$ is a compact, oriented submanifold, then if $\rho$ is a closed $n$-form on $N$,

$$
\int_{N} \rho=\int_{M} \tilde{\rho} \wedge \mathscr{D}([N])
$$

for any closed $n$-form $\tilde{\rho}$ on $M$ whose restriction to $N$ is $\rho$.

Consider the product space $\mathbf{P}^{n} \times \mathbf{P}^{n}$ with projections $\pi_{i}, i=1,2$, onto each factor. Let $\omega_{i}=\pi_{i}^{*} \omega, i=1,2$, be the pullback of the Kähler form to the product. Letting $\Delta \subset \mathbf{P}^{n} \times \mathbf{P}^{n}$ denote the diagonal, we have, for any positive integer $\lambda$, $\left.\frac{1}{2}\left(\omega_{1}^{\lambda}+\omega_{2}^{\lambda}\right)\right|_{\Delta}=\omega^{\lambda}$, where $\omega$ is the Kähler form on $\Delta \cong \mathbf{P}^{n}$. The usual diagonal trick of algebraic geometry can now be applied. Set $\lambda=p+q-n-k-l$; since $(X \times Y) \cap \Delta \cong X \cap Y$,

$$
\begin{aligned}
\int_{X \cap Y} \phi \wedge \psi \wedge \omega^{\lambda} & =\frac{1}{2} \int_{(X \times Y) \cap \Delta} \pi_{1}^{*} \phi \wedge \pi_{2}^{*} \psi \wedge\left(\omega_{1}^{\lambda}+\omega_{2}^{\lambda}\right) \\
& =\frac{1}{2} \int_{X \times Y} \pi_{1}^{*} \phi \wedge \pi_{2}^{*} \psi \wedge\left(\omega_{1}^{\lambda}+\omega_{2}^{\lambda}\right) \wedge \mathcal{D}(\Delta) \\
& =\frac{1}{2} \int_{X \times Y} \pi_{1}^{*} \phi \wedge \pi_{2}^{*} \psi \wedge\left(\omega_{1}^{\lambda}+\omega_{2}^{\lambda}\right) \wedge \sum_{\mu=0}^{n} \omega_{1}^{\mu} \wedge \omega_{2}^{n-\mu} \\
& =\frac{1}{2} \sum_{\mu=0}^{n} \int_{X} \phi \wedge \omega^{\lambda+\mu} \int_{Y} \psi \wedge \omega^{n-\mu}+\int_{X} \phi \wedge \omega^{\mu} \int_{Y} \psi \wedge \omega^{\lambda+n-\mu} \\
& =\int_{X} \phi \wedge \omega^{p-k} \int_{Y} \psi \wedge \omega^{q-1}, \text { as required. }
\end{aligned}
$$

We remark that according to the first paragraph of the proof, the result is independent of our choice of form whose restriction to the diagonal is $\omega^{\lambda}$. 
Combining Lemma (4.2) with (4.1) gives

(4.3) Proposition. Let $X^{p}, Y^{q} \subset \mathbf{P}^{n}$ be nonsingular algebraic varieties intersecting transversely. Then for $j=0, \ldots, p+q-n$,

$$
\begin{aligned}
\int_{X \cap Y} c_{j}\left(\Omega_{X \cap Y}\right) \wedge \omega^{p+q-n-j} & \\
= & \sum_{\substack{i, l \\
0<i+l<j}}(-1)^{j-i-l}\left(\begin{array}{c}
n+j-i-l \\
n
\end{array}\right) \int_{X} c_{i}\left(\Omega_{X}\right) \wedge \omega^{p-i} \int_{Y} c_{l}\left(\Omega_{Y}\right) \wedge \omega^{q-l} .
\end{aligned}
$$

To put this in the framework of a kinematic formula, we consider the group $U(n+1)$ of motions of $\mathbf{P}^{n}$. For generic $g \in U(n+1), X$ will be transverse to $g Y$ and thus Proposition (4.3) is valid generically. Let $d g$ be the invariant Haar measure on $U(n+1)$. We can then recast Proposition (4.3) in the integrated form

$$
\begin{gathered}
\int_{U(n+1)}\left(\int_{X \cap g Y} c_{j}\left(\Omega_{X \cap g Y}\right) \wedge \omega^{p+q-n-j}\right) d g \\
=\sum_{\substack{i, l \\
0<i+l<j}} a_{i l} \int_{X} c_{i}\left(\Omega_{X}\right) \wedge \omega^{p-i} \int_{Y} c_{l}\left(\Omega_{Y}\right) \wedge \omega^{q-l}, \\
a_{i l}=\operatorname{vol}(U(n+1)) \cdot(-1)^{j}\left(\begin{array}{c}
n+j-i-l \\
n
\end{array}\right) .
\end{gathered}
$$

The rest of our work will be devoted to showing that (4.4) holds locally as well. One may think of this result as a local, integrated generalization of classical Bezout theorems and adjunction formulas in algebraic geometry (and, of course, as a complexification of the corresponding kinematic formulas [6] for Riemannian manifolds).

In analogy with the way we proceeded in the linear case, we might seek a universal formula in the following manner. We define an incidence correspondence $I^{\prime} \subset \mathbf{G}(0, p, n) \times \mathbf{G}(0, q, n) \times \mathbf{G}(0, p+q-n, n) \times U(n+1)$ by

$$
I^{\prime}=\left\{\left((z, T),\left(z^{\prime}, L\right),\left(z^{\prime \prime}, S\right), g\right): z=z^{\prime \prime}=g z^{\prime}, S \subset T \cap g L\right\},
$$

together with projections:

$$
\begin{array}{ccc}
\pi_{1}^{\prime} \swarrow & I^{\prime} & \\
\mathbf{G}(0, p, n) \times \mathbf{G}(0, q, n) & & \mathbf{G}(0, p+q-n, n) \times U(n+1)
\end{array}
$$

Note that $U(n+1)$ acts naturally-by right translation on the pointed Grassmannians and by the adjoint action on itself-and leaves $I^{\prime}$ invariant. Let $E^{\prime \prime}=$ $E_{p+q-n+1}, E=E_{p+1}, E^{\prime}=E_{q+1}$ and $\omega^{\prime \prime}, \omega, \omega^{\prime}$ denote the universal bundles and Kähler forms on $\mathbf{G}(0, p+q-n, n), \mathbf{G}(0, p, n)$ and $\mathbf{G}(0, q, n)$ respectively. We would like to see that $\pi_{1 *}^{\prime} \pi_{2}^{\prime *}\left(c_{j}\left(\Omega_{E^{\prime \prime}}\right) \wedge \omega^{\prime p+q-n-j} \wedge d g\right)$ is-at least modulo an appropriate differential ideal-a polynomial with constant coefficients in Chern forms of $\Omega_{E}$ and $\Omega_{E^{\prime}}$ and the Kähler forms $\omega$ and $\omega^{\prime}$.

One might suspect-and quite rightly so-that matters will be further complicated by the fact that $\mathbf{G}(0, p, n) \times \mathbf{G}(0, q, n)$ is not homogeneous with respect to the 
action of $U(n+1)$. In particular, there is no reason that the only invariant forms on the product space which appear in the result should be generated by products of invariant forms. It is a priori not at all clear, for example, that the "angle" between the $p$-plane and $q$-plane will not appear, insofar as it is certainly an invariant function. The deus ex machina is, at this point, the introduction of moving frames. By lifting the diagram (4.5) to the associated frame bundles, we are able to integrate over $U(n+1)$ piecemeal, thereby isolating the group actions. Because this proof is more involved than preceding ones, we give the main steps of the proof here and postpone a few technicalities to the next section.

We start with some new definitions and notation. Let

$$
\operatorname{St}(k, N)=U(N) / U(N-k)
$$

be the Stiefel manifold of unitary $k$-frames in $\mathbf{C}^{N}$. Note that $\operatorname{St}(k, N)$ fibers over $G(k, N)$ with fiber $U(k)$; this is the principal $U(k)$-bundle associated to the universal subbundle $E_{k}$ on $G(k, N)$. Working in $\mathbf{P}^{n}$, we define $\operatorname{St}(k, n)=$ $\operatorname{St}(k+1, n+1)$. Given a frame $f=\left(f_{0}, \ldots, f_{k}\right) \in \operatorname{St}(k, n)$, we let $\left[f_{0}\right] \overline{\operatorname{den}}$ tete the projection of $f_{0} \in \mathbf{C}^{n+1}$ to $\mathbf{P}^{n}$. We now define various incidence correspondences in terms of the Stiefel manifolds. Set

$$
\begin{aligned}
\mathbf{G}(0, d, n) & =\left\{(z, Q) \in \mathbf{P}^{n} \times \mathbf{G}(d, n): z \in Q\right\} \quad \text { (as before), } \\
\underline{\mathrm{St}}(0, k, n) & =\left\{(z, f) \in \mathbf{P}^{n} \times \underline{\mathrm{St}}(k, n): z=\left[f_{0}\right]\right\}, \\
\underline{\mathrm{St}}(0, k, d, n) & =\left\{(z, f, Q) \in \mathbf{P}^{n} \times \underline{\mathrm{St}}(k, n) \times \mathbf{G}(d, n): z=\left[f_{0}\right],\right.
\end{aligned}
$$

$f$ is a frame for a subspace of $Q\}$.

We now set $k=p+q-n$.

Armed with this notation, define the incidence correspondence

$$
\begin{gathered}
I \subset \underline{\mathrm{St}}(0, k, p, n) \times \underline{\mathrm{St}}(0, k, q, n) \times \underline{\mathrm{St}}(0, k, n) \times U(n+1), \\
I=\left\{\left((z, f, T),\left(z^{\prime}, f^{\prime}, L\right),\left(z^{\prime \prime}, f^{\prime \prime}\right), g\right): z=z^{\prime \prime}=g z^{\prime}, f=f^{\prime \prime}=g f^{\prime}\right\} .
\end{gathered}
$$

One may check that $I$ is invariant under the obvious action of $U(n+1)$. Here, then, is the giant diagram which will be the subject of our attention.

$$
\begin{aligned}
& \pi_{2} \quad I \quad \pi^{\pi_{3}}
\end{aligned}
$$

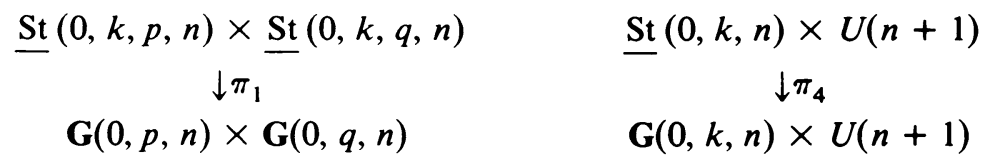

For the record, we note that

$$
\begin{aligned}
& \text { fiber } \pi_{4} \cong U(1) \times U(k), \quad \text { fiber } \pi_{2} \cong U(n-k), \\
& \text { fiber } \pi_{1} \cong U(1) \times \operatorname{St}(k, p) \times U(1) \times \operatorname{St}(k, q) .
\end{aligned}
$$

Thus, if we start with a form $\phi$ of degree $2 k$ on $\mathbf{G}(0, k, n)$ and let

$$
\Phi=\left(\pi_{4 *}\right)^{-1}(\phi \wedge d g)=\pi_{4}^{*}(\phi \wedge d g) \wedge d h,
$$


where $d h$ is the volume form of the fiber of $\pi_{4}$, then $\Phi$ has degree $2 k+(n+1)^{2}+$ $1+k^{2}$, and therefore $\pi_{1 *} \pi_{2 *} \pi_{3}^{*} \Phi$ is a form of degree

$$
\begin{aligned}
{\left[2 k+(n+1)^{2}+\right.} & \left.\left(k^{2}+1\right)\right]-\left[(n-k)^{2}+2+k(2 p-k+2 q-k)\right] \\
& =2(k+n)=2(p+q) .
\end{aligned}
$$

Notation. Given a form $\phi$ on $\mathbf{G}(0, k, n)$, let $\tilde{\phi}=\pi_{1 *} \pi_{2 *} \pi_{3}^{*}\left(\left(\pi_{4 *}\right)^{-1}(\phi \wedge d g)\right)$ denote the form on $\mathbf{G}(0, p, n) \times \mathbf{G}(0, q, n)$ obtained in this manner.

For the remainder of our work, we shall use the following ranges of indices.

Put $k=p+q-n$,

$$
\begin{array}{ll}
0 \leqslant A, B, C \leqslant n, & 0 \leqslant \alpha, \beta \leqslant k, \\
1 \leqslant a, b, c \leqslant n, & 1 \leqslant \gamma, \delta \leqslant k, \\
k+1 \leqslant i, j \leqslant n, & p+1 \leqslant \lambda, \mu \leqslant n, \\
k+1 \leqslant r, s \leqslant p, & q+1 \leqslant \rho, \sigma \leqslant n, \\
k+1 \leqslant l \leqslant q, & 0 \leqslant \nu, \tau \leqslant p, \quad 0 \leqslant \eta, \zeta \leqslant q .
\end{array}
$$

In keeping with our convention, we use unprimed symbols for objects (e.g., Kähler form, bundles, curvature forms) on $\mathbf{G}(0, p, n)$, primed on $\mathbf{G}(0, q, n)$, and doubleprimed on $\mathbf{G}(0, k, n)$. In particular, $E, E^{\prime}, E^{\prime \prime}, \Omega, \Omega^{\prime}, \Omega^{\prime \prime}$, and $\omega, \omega^{\prime}, \omega^{\prime \prime}$ denote the universal bundles, their curvatures, and the Kähler forms (pulled back from $\mathbf{P}^{n}$ ) of these three spaces respectively.

Having concocted the elaborate fibration (4.6) and established the tiers of notation, we proceed to the proof of an appropriate universal formula. Various technical points will be deferred to the end of this section, at which point we solemnly promise every index will appear.

(4.8) TheORem. Let $0 \leqslant j \leqslant k$, and let $\phi=c_{j}\left(\Omega^{\prime \prime}\right) \wedge \omega^{\prime \prime k-j}$. Modulo the differential ideal $G=\left(\omega^{\lambda}, \omega^{\bar{\lambda}}, \omega^{\prime \rho}, \omega^{\prime \bar{\rho}}\right), \tilde{\phi}=P\left(\Omega, \Omega^{\prime}, \omega, \omega^{\prime}\right)$ is a polynomial in the Chern forms of $\Omega$ and $\Omega^{\prime}$ and the Kähler forms $\omega, \omega^{\prime}$ with constant coefficients and of total degree $2(p+q)$.

In the course of the proof we shall use the following fact whose proof is similar to arguments we have already given.

(4.9) FACT. $\omega_{\nu \bar{\lambda}}, \omega_{\alpha \bar{\nu}}$ and their conjugates give a basis for the 1-forms on $\underline{\mathrm{St}}(0, k, p, n)$.

Proof of Theorem (4.8). Step 1. First we must analyze $\pi_{2 *} \pi_{3}^{*} \Phi$ on $\operatorname{St}(0, k, p, n)$ $\times \underline{\operatorname{St}}(0, k, q, n)$, where $\Phi=\left(\pi_{4 *}\right)^{-1}(\phi \wedge d g)$. By Fact (4.9) all forms on $\overline{\operatorname{St}}(0, k, p, n)$ $\times \overline{\mathrm{St}}(0, k, q, n)$ are locally expressible in terms of $\omega_{\nu \bar{\lambda}}, \omega_{\alpha \bar{\nu}}, \omega_{\zeta \bar{\rho}}^{\prime}, \omega_{\alpha \bar{\zeta}}^{\prime}$, and their conjugates (recall $\omega^{A}=\omega_{0 \bar{A}}$ ).

While a priori $\pi_{2 *} \pi_{3}^{*} \Phi$ is some expression involving all the 1 -forms we have specified, the fact that it is invariant under fiber $\pi_{2} \cong U(n-k)$, and in particular under the subgroup $U(n-p) \times U(n-q) \subset U(n-k)$, is very telling. Specifically, the transformations,

$$
\omega_{\nu \bar{\lambda}}^{*}=\sum g_{\mu \bar{\lambda}} \omega_{\nu \bar{\mu}}, \quad \omega_{\zeta \bar{\rho}}^{\prime *}=\sum g_{\sigma \bar{\rho}} \omega_{\zeta \bar{\sigma}}^{\prime},
$$

for $\left(g_{\mu \bar{\lambda}}\right) \in U(n-p),\left(g_{\sigma \bar{\rho}}\right) \in U(n-q)$ must leave the form $\pi_{2 *} \pi_{3}^{*} \Phi$ invariant. The discussion of the Appendix (cf., for example, the proof of Theorem (1.28)) then 
applies and we infer that the form must be a polynomial in $\Omega_{\nu \bar{\tau}}=\sum \omega_{\nu \bar{\lambda}} \wedge \omega_{\lambda \bar{\tau}}$ and $\Omega_{\eta \bar{\zeta}}^{\prime}=\sum \omega_{\eta \bar{\rho}}^{\prime} \wedge \omega_{\rho \bar{\zeta}}^{\prime}$ with coefficients expressions involving $\omega_{\alpha \bar{\nu}}$ and $\omega_{\alpha \bar{\zeta}}^{\prime}-$ not constants, since the group does not act transitively on $\underline{\mathrm{St}}(0, k, p, n) \times \underline{\mathrm{St}}(0, k, q, n)$. Notice that included in this list are expressions such as $\Omega_{0 \overline{0}}=\sum \omega^{\lambda} \wedge \omega^{\bar{\lambda}}, \Omega_{0_{\bar{\tau}}}=$ $\sum \omega^{\lambda} \wedge \omega_{\lambda \bar{\tau}}$, etc., all of which are zero modulo 9 .

Step 2. Next we claim that integrating over the fiber of $\pi_{1}$ gives a form which is invariant under $(U(1) \times U(p)) \times(U(1) \times U(q))$. To see this, we introduce an auxiliary fibration:

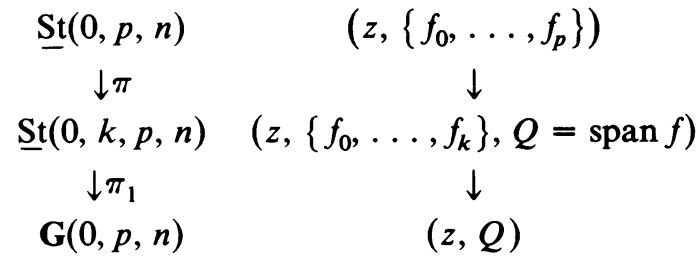

For any form $\psi$ on $\underline{\operatorname{St}}(0, k, p, n),\left(\pi_{1}^{\circ} \pi_{*}\left(\pi_{*}^{-1} \psi\right)\right.$ is $(U(1) \times U(p))$-invariant, and hence so is $\pi_{1 *} \psi$.

Now repeating the earlier arguments verbatim shows that $\pi_{1 *} \pi_{2 *}\left(\pi_{3}^{*} \phi\right)$ must be modulo $g$ a polynomial in Chern forms of $\Omega, \Omega^{\prime}$ and in the Kähler forms $\omega, \omega^{\prime}$. The coefficients are smooth functions on $\mathbf{G}(0, p, n) \times \mathbf{G}(0, q, n)$ which are invariant under the $U(n+1)$-action, i.e., which are constant on orbits. (Note, however, that a priori they need not be constants.)

Step 3. At this point in the analysis, we must resort to an argument which was not required previously. Since $\phi$ is closed, by (A.2) so then is $\tilde{\phi}$. Now an elementary (but crucially important!) computation of exterior derivatives and type considerations show that the coefficient functions must in fact be constants. The proof is now complete. We reiterate that at this point a cohomological argument is not sufficient, since there are likely nontrivial exact invariant forms in this situation.

We remark before proceeding that by considering frames here we were able to separate the action of $U(n+1)$ on the two spaces and hence reduce the argument to essentially the linear case. We now repeat earlier reasoning, somewhat more sketchily, to prove the main result.

(4.10) Theorem (Kinematic formula in $\mathbf{P}^{n}$ ). Let $X^{p}, Y^{q} \subset \mathbf{P}^{n}$ be complex submanifolds (perhaps with boundary). For $j=0, \ldots, k=p+q-n$,

$$
\begin{aligned}
\int_{U(n+1)}\left(\int_{X \cap g Y} c_{j}\left(\Omega_{X \cap g Y}\right) \wedge \omega^{k-j}\right) d g \\
=\sum_{\substack{i, l \\
0<i+l<j}} a_{i l} \int_{X} c_{i}\left(\Omega_{X}\right) \wedge \omega^{p-i} \int_{Y} c_{l}\left(\Omega_{Y}\right) \wedge \omega^{q-l} \\
a_{i l}=\operatorname{vol}(U(n+1)) \cdot(-1)^{j}\left(\begin{array}{c}
n+j-i-l \\
n
\end{array}\right)
\end{aligned}
$$

whenever both sides make sense. 
Proof. The proof is a straightforward computation using the universal formula we have just derived. Let $\gamma: X \times Y \rightarrow \mathbf{G}(0, p, n) \times \mathbf{G}(0, q, n)$ be the product pointed Gauss mapping. Let $\phi=c_{j}\left(\Omega^{\prime \prime}\right) \wedge \omega^{\prime \prime k-j}$ on $\mathbf{G}(0, k, n)$ and compute $\tilde{\phi}$. From Theorem (4.8) we deduce that this is a polynomial $P_{j}\left(\Omega, \Omega^{\prime}, \omega, \omega^{\prime}\right)$ with constant coefficients whose pullback under $\gamma$ is $P_{j}\left(\tilde{\Omega}_{X}, \tilde{\Omega}_{Y}, \omega_{X}, \omega_{Y}\right)$. By earlier methods, this then gives a universal polynomial $P_{j}^{\prime}\left(\Omega_{X}, \Omega_{Y}, \omega_{X}, \omega_{Y}\right)$ of total degree $2(p+q)$ on $X \times Y$.

Let $H=U(1) \times U(k)$ be the fiber of $\pi_{4}$, and $d h$ the Haar measure on $H$. Repeating the second step in the proof of Theorem (3.8) leads to

$$
\begin{aligned}
\int_{X \times Y} \gamma^{*} \tilde{\phi} & =\int_{\gamma(X \times Y)} \tilde{\phi}=\int_{\left(\pi_{1} \pi_{2}\right)^{-1}(\gamma(X \times Y))} \pi_{3}^{*}\left(\pi_{4 *}\right)^{-1}(\phi \wedge d g) \\
& =\int_{Z}\left(\pi_{4 *}\right)^{-1}(\phi \wedge d g),
\end{aligned}
$$

where $Z=\pi_{3}\left(\left(\pi_{1} \circ \pi_{2}\right)^{-1}(\gamma(X \times Y))\right)$ and we have used the fact that $\left.\pi_{3}\right|_{\pi_{3}^{-1}(Z)}$ is a diffeomorphism,

$$
\begin{aligned}
& =\int_{\pi_{4}(Z)} \phi \wedge d g \int_{H} d H \\
& =\operatorname{vol}(H) \int_{\substack{g \in U(n+1) \\
X \mp Y}}\left(\int_{X \cap g Y} c_{j}\left(\Omega_{X \cap g Y}\right) \wedge \omega^{k-j}\right) d g .
\end{aligned}
$$

As in the linear case, one reinterprets the latter integral in terms of the usual tangent bundle, computes the constants from the algebraic case, and is virtually done.

There is, however, a reasonably serious issue remaining. While the set of $g \in U(n+1)$ for which $X$ and $g Y$ fail to meet transversely is indeed of measure zero, we must insure convergence of the integrals. We deal carefully with this matter next.

In passing, we observe that it is by now no surprise that a slightly stronger result is valid.

(4.12) THEOREM. Let $P\left(\Omega^{\prime \prime}, \omega^{\prime \prime}\right)$ be a polynomial of total degree

$$
2 k=2(p+q-n)
$$

in Chern forms $c_{i}\left(\Omega^{\prime \prime}\right)$ and the Kähler form $\omega^{\prime \prime}$. Then there is canonically associated to $P$ a polynomial $Q\left(\Omega, \Omega^{\prime}, \omega, \omega^{\prime}\right)$ of total degree $2(p+q)$ so that

$$
\int_{U(n+1)}\left(\int_{X \cap g Y} P\left(\Omega_{X \cap g Y}, \omega\right)\right) d g=\int_{X \times Y} Q\left(\Omega_{X}, \Omega_{Y}, \omega_{X}, \omega_{Y}\right)
$$

for any submanifolds $X^{p}, Y^{q} \subset \mathbf{P}^{n}$ (with boundary), for which both sides make sense.

As before, $Q$ can in principle be computed from the algebraic case.

The most obvious geometric invariant possessed by a $p$-plane and a $q$-plane in $\mathbf{P}^{n}$ is one to which we have already alluded several times, the "angle" they define. This angle becomes zero as the planes meet nontransversely. One might expect this fact 
to cause trouble in integrals such as (4.11), and we want to deal effectively with this question here. To ascertain the convergence of the integrals, we begin with a specific change of variables theorem on the incidence correspondence $I$ which will allow us to integrate over the fiber of $\pi_{2}$ "explicitly". Then we shall need a generalized Meusnier's theorem relating the curvature forms $\Omega, \Omega^{\prime}, \Omega^{\prime \prime}$ on $I$ (i.e., relating the curvature of $X \cap Y$ to that of $X$ and $Y$ ). Then we shall address the question of convergence.

We use the ranges of indices (4.7), and before beginning have one last bout with establishing notation. Let $\mathbf{a}=\left\{a_{A}\right\}$ be a fixed frame field for $\mathbf{P}^{n}$, i.e., a fixed basis for $\mathbf{C}^{n+1}, \mathbf{a}^{\prime}=\left\{a_{A}^{\prime}\right\}$ a moving frame associated to the action of $G=U(n+1)$ on $\mathbf{P}^{n}$; more precisely, $a_{A}^{\prime}$ are $\mathbf{C}^{n+1}$-valued functions on $G$ given by $\mathbf{a}^{\prime}=g \mathbf{a}$. We denote by $d$ and $d^{\prime}$ differentiation with respect to the frames a, $\mathbf{a}^{\prime}$ respectively. Observe that $d=d^{\prime}$ on functions, and, by definition, $d a_{A}=d^{\prime} a_{A}^{\prime}=0$. Let $e_{A}, e_{A}^{\prime}$ be unitary frames adapted to $\mathbf{G}(0, p, n)$ and $\mathbf{G}(0, q, n)$ respectively, normalized so that $e_{\alpha}=e_{\alpha}^{\prime}$ on $I(\alpha=0, \ldots, k)$, assuming transverse intersection. Define

$$
\Delta=\operatorname{det}\left(\left(e_{\lambda}, e_{l}^{\prime}\right)\right)=\operatorname{det}\left(\left(e_{r}, e_{\rho}^{\prime}\right)\right)
$$

note $\Delta$ is nonzero precisely when $T=e_{0} \wedge \cdots \wedge e_{p}$ and $L=e_{0}^{\prime} \wedge \cdots \wedge e_{q}^{\prime}$ meet transversely. We may think of $\Delta$ as the function on $\mathbf{G}(0, p, n) \times \mathbf{G}(0, q, n)$ giving the angle between the two planes.

One may view the following forms as being defined on the incidence correspondence $I$.

$$
\begin{aligned}
& d g=\text { Haar measure on } G=U(n+1)=\Lambda\left(d a_{A}^{\prime}, a_{B}^{\prime}\right) \text {, } \\
& d h=\text { Haar measure on } H=U(1) \times U(k)=d \theta \wedge \Lambda\left(d e_{\gamma}, e_{\delta}\right) \text {, } \\
& d k=\text { Haar measure on } K=U(n-k) \text {, } \\
& \psi_{1}=\omega^{1} \wedge \cdots \wedge \omega^{p} \wedge \omega^{\overline{1}} \wedge \cdots \wedge \omega^{\bar{p}} \wedge \omega^{\prime 1} \wedge \cdots \wedge \omega^{\prime q} \wedge \omega^{\prime 1} \wedge \cdots \wedge \omega^{\prime \bar{q}} \text {, } \\
& \psi_{2}=\text { volume element on } \operatorname{St}(k, p)=\Lambda\left(d e_{\gamma}, e_{\delta}\right) \wedge\left(d e_{\gamma}, e_{s}\right) \wedge \overline{\left(d e_{\gamma}, e_{s}\right)} \text {, } \\
& \psi_{3}=\text { volume element on } \operatorname{St}(k, q)=\Lambda\left(d^{\prime} e_{\gamma}, e_{\delta}\right) \wedge\left(d^{\prime} e_{\gamma}^{\prime}, e_{l}^{\prime}\right) \wedge \overline{\left(d^{\prime} e_{\gamma}^{\prime}, e_{l}^{\prime}\right)} \text {, } \\
& \phi=\Lambda\left(d e_{0}, e_{\gamma}\right) \wedge \overline{\left(d e_{0}, e_{\gamma}\right)} \text {, } \\
& d \theta=d^{\prime} \theta=\text { volume element on } S^{1} \text {. }
\end{aligned}
$$

Proposition (ChANge of VARIABles formula). Modulo the differential ideal $g=\left(\omega^{\lambda}, \omega^{\bar{\lambda}}, \omega^{\rho}, \omega^{\prime \bar{\rho}}\right)$, we have the following equality on the incidence correspondence I:

$$
\phi \wedge d g \wedge d h \wedge d \theta= \pm\left|\Delta^{k+1}\right|^{2} \psi_{1} \wedge \psi_{2} \wedge \psi_{3} \wedge d k \wedge d \theta \wedge d^{\prime} \theta
$$

PRoof. We follow Chern [6] in introducing the operator $d-d^{\prime}$. If $e_{A}^{\prime}=\Sigma u_{A \bar{B}} a_{B}^{\prime}$, where $\left(u_{A \bar{B}}\right)$ is a unitary matrix, then

$$
\left(d-d^{\prime}\right) e_{A}^{\prime}=\sum\left(d u_{A \bar{B}}-d^{\prime} u_{A \bar{B}}\right) a_{B}^{\prime}+u_{A \bar{B}} d a_{B}^{\prime}=\sum u_{A \bar{B}} d a_{B}^{\prime} .
$$

Since $\left(u_{A \bar{B}}\right)$ is unitary, it follows that

$$
d g=\Lambda\left(d a_{A}^{\prime}, a_{B}^{\prime}\right)=\Lambda\left(\left(d-d^{\prime}\right) e_{A}^{\prime}, e_{B}^{\prime}\right) .
$$

Similarly, the Haar measure for the isotropy subgroup $K$ is

$$
d k=\Lambda\left(\left(d-d^{\prime}\right) e_{i}^{\prime}, e_{j}^{\prime}\right) .
$$

We delete $d \theta$ from both sides of (4.14) and rewrite the left-hand side. We number 
certain steps in the following derivation on which we will comment briefly afterwards.

$$
\begin{aligned}
& \phi \wedge d g \wedge d h=\Lambda\left(d e_{0}, e_{\gamma}\right) \wedge \overline{\left(d e_{0}, e_{\gamma}\right)} \wedge\left(\left(d-d^{\prime}\right) e_{A}^{\prime}, e_{B}^{\prime}\right) \wedge\left(d e_{\gamma}, e_{\delta}\right) \\
& = \pm \Lambda\left(d e_{0}, e_{\gamma}\right) \wedge \overline{\left(d e_{0}, e_{\gamma}\right)} \wedge\left(\left(d-d^{\prime}\right) e_{i}^{\prime}, e_{j}^{\prime}\right) \wedge\left(\left(d-d^{\prime}\right) e_{0}, e_{0}\right) \\
& \wedge\left(\left(d-d^{\prime}\right) e_{0}, e_{a}^{\prime}\right) \wedge\left(\left(d-d^{\prime}\right) e_{a}^{\prime}, e_{0}\right) \wedge\left(\left(d-d^{\prime}\right) e_{\gamma}, e_{\delta}\right) \\
& \wedge\left(\left(d-d^{\prime}\right) e_{\gamma}, e_{i}^{\prime}\right) \wedge\left(\left(d-d^{\prime}\right) e_{i}^{\prime}, e_{\gamma}\right) \wedge\left(d e_{\gamma}, e_{\delta}\right) \\
& \stackrel{(1)}{=} \pm\left[d k \wedge d^{\prime} \theta \wedge \Lambda\left(d e_{\gamma}, e_{\delta}\right) \wedge\left(d^{\prime} e_{\gamma}, e_{\delta}\right) \wedge\left(d e_{0}, e_{\gamma}\right)\right. \\
& \left.\wedge \overline{\left(d e_{0}, e_{\gamma}\right)} \wedge\left(d^{\prime} e_{0}, e_{\gamma}\right) \wedge \overline{\left(d^{\prime} e_{0}, e_{\gamma}\right)}\right] \\
& \wedge \Lambda\left(\left(d-d^{\prime}\right) e_{0}, e_{l}^{\prime}\right) \wedge\left(\left(d-d^{\prime}\right) e_{l}^{\prime}, e_{0}\right) \\
& \wedge\left(\left(d-d^{\prime}\right) e_{\gamma}, e_{l}^{\prime}\right) \wedge\left(\left(d-d^{\prime}\right) e_{l}^{\prime}, e_{\gamma}\right) \\
& \wedge\left(\left(d-d^{\prime}\right) e_{\alpha}, e_{\rho}^{\prime}\right) \wedge\left(\left(d-d^{\prime}\right) e_{\rho}^{\prime}, e_{\alpha}\right)^{-} \\
& \stackrel{(2)}{=} \pm[\cdots] \wedge \Lambda\left(d e_{\alpha}, e_{\rho}^{\prime}\right) \wedge\left(d e_{\rho}^{\prime}, e_{\alpha}\right) \\
& \wedge\left[\Lambda\left(\left(d-d^{\prime}\right) e_{0}, e_{l}^{\prime}\right) \wedge\left(\left(d-d^{\prime}\right) e_{l}^{\prime}, e_{0}\right)\right. \\
& \left.\wedge\left(\left(d-d^{\prime}\right) e_{\gamma}, e_{l}^{\prime}\right) \wedge\left(\left(d-d^{\prime}\right) e_{l}^{\prime}, e_{\gamma}\right)\right] \\
& \stackrel{(3)}{=} \pm[\cdots]\left|\Delta^{k+1}\right|^{2} \wedge \Lambda\left(d e_{\alpha}, e_{r}\right) \wedge \overline{\left(d e_{\alpha}, e_{r}\right)} \wedge[\cdots] \\
& \stackrel{(4)}{=} \pm\left|\Delta^{k+1}\right|^{2} d k \wedge d^{\prime} \theta \wedge \Lambda\left(d e_{\gamma}, e_{\delta}\right) \wedge\left(d^{\prime} e_{\gamma}, e_{\delta}\right) \wedge\left(d e_{0}, e_{\gamma}\right) \\
& \wedge \overline{\left(d e_{0}, e_{\gamma}\right)} \wedge\left(d e_{0}, e_{r}\right) \wedge \overline{\left(d e_{0}, e_{r}\right)} \wedge\left(d^{\prime} e_{0}, e_{\gamma}\right) \wedge \overline{\left(d^{\prime} e_{0}, e_{\gamma}\right)} \\
& \wedge\left(d e_{\gamma}, e_{r}\right) \wedge \overline{\left(d e_{\gamma}, e_{r}\right)} \wedge\left(d^{\prime} e_{0}, e_{l}^{\prime}\right) \wedge \overline{\left(d^{\prime} e_{0}, e_{l}^{\prime}\right)} \\
& \wedge\left(\left(d-d^{\prime}\right) e_{\gamma}, e_{l}^{\prime}\right) \wedge\left(\left(d-d^{\prime}\right) e_{l}^{\prime}, e_{\gamma}\right) \\
& \stackrel{(5)}{=} \pm\left|\Delta^{k+1}\right|^{2} d k \wedge d^{\prime} \theta \wedge \Lambda\left\{\left(d e_{0}, e_{\gamma}\right) \wedge \overline{\left(d e_{0}, e_{\gamma}\right)} \wedge\left(d e_{0}, e_{r}\right) \wedge \overline{\left(d e_{0}, e_{r}\right)}\right\} \\
& \wedge\left\{\left(d^{\prime} e_{0}, e_{\gamma}\right) \wedge \overline{\left(d^{\prime} e_{0}, e_{\gamma}\right)} \wedge\left(d^{\prime} e_{0}, e_{l}^{\prime}\right) \wedge \overline{\left(d^{\prime} e_{0}, e_{l}^{\prime}\right)}\right\} \\
& \wedge\left\{\left(d e_{\gamma}, e_{\delta}\right) \wedge\left(d e_{\gamma}, e_{r}\right) \wedge \overline{\left(d e_{\gamma}, e_{r}\right)}\right\} \\
& \wedge\left\{\left(d^{\prime} e_{\gamma}, e_{\delta}\right) \wedge\left(d^{\prime} e_{\gamma}, e_{l}^{\prime}\right) \wedge \overline{\left(d^{\prime} e_{\gamma}, e_{l}^{\prime}\right)}\right\} \\
& = \pm\left|\Delta^{k+1}\right|^{2} d k \wedge d^{\prime} \theta \wedge \psi_{1} \wedge \psi_{2} \wedge \psi_{3}, \quad \text { as required. }
\end{aligned}
$$

The various steps are valid:

(1) modulo $d \boldsymbol{\theta}$ (introduced by the last two terms);

(2) modulo 9 , since $\left(d^{\prime} e_{\alpha}, e_{\rho}^{\prime}\right)=-\overline{\left(d^{\prime} e_{\rho}^{\prime}, e_{\alpha}\right)} \equiv 0 \bmod \omega^{\prime \alpha}, \omega^{\prime \rho}$;

(3) using (4.13);

(4) insofar as $\left(d e_{0}, e_{l}^{\prime}\right)$ terms do not enter $\bmod \omega^{\alpha}, \omega^{r}$ since we already have the requisite number of terms $\left(d e_{0}, e_{A}\right)$;

(5) because, similarly, $\left(d e_{\gamma}, e_{l}^{\prime}\right)$ do not enter since $\left(d e_{\gamma}, e_{l}\right)=0$ modulo $\omega^{A}, \omega^{\bar{A}}$. The proof is complete. 
In order to deal with the convergence of the integrals (4.11) near points of nontransverse intersection, we must study the behavior of $\Psi=c_{j}\left(\Omega^{\prime \prime}\right) \wedge \omega^{\prime \prime k-j} \wedge$ $d g \wedge d h \wedge d \theta=\pi_{3}^{*}\left(\left(\pi_{4 *}\right)^{-1} \phi \wedge d g\right)$ on $I$. In particular, one must watch the growth of $c_{j}\left(\Omega^{\prime \prime}\right)$. In this light, we would like an explicit formula for $\Omega^{\prime \prime}$ in terms of $\omega_{\alpha \bar{\lambda}}, \omega_{\alpha \bar{\rho}}^{\prime}$ and their conjugates.

(4.15) Proposition (Meusnier theOrem). On the incidence correspondence $I, \Omega^{\prime \prime}$ can be written as a polynomial in $\omega_{\alpha \bar{\lambda}}, \omega_{\alpha \bar{\rho}}^{\prime}$ and their conjugates, and the coefficients are $O\left(|\Delta|^{-2}\right)$ near $\Delta=0$.

Proof. We put

$$
e_{\rho}^{\prime}=\sum u_{\rho \bar{s}} e_{s}+\sum u_{\rho \bar{\lambda}} e_{\lambda}
$$

where $\left(u_{\rho \bar{s}}\right)$ is invertible precisely when $T=e_{0} \wedge \cdots \wedge e_{p}$ and $g L=e_{0}^{\prime}$ $\wedge \cdots \wedge e_{q}^{\prime}$ meet transversely. In this event, we put $\left(v_{r \bar{\sigma}}\right)=\left(u_{\rho \bar{s}}\right)^{-1}$. Using (4.16) we solve for $e_{r}$ :

$$
e_{r}=\sum v_{r \bar{\rho}} e_{\rho}^{\prime}-\sum v_{r \bar{\rho}} u_{\rho \bar{\lambda}} e_{\lambda}=\sum v_{r \bar{\rho}} e_{\rho}^{\prime}-\sum v_{r \bar{\lambda}} e_{\lambda},
$$

where we put $v_{r \bar{\lambda}}=\sum v_{r \bar{\rho}} u_{\rho \bar{\lambda}}$. Therefore,

$$
\omega_{\alpha \bar{r}}=\sum \bar{v}_{r \bar{\rho}} \omega_{\alpha \bar{\rho}}^{\prime}-\sum \bar{v}_{r \bar{\lambda}} \omega_{\alpha \bar{\lambda}}
$$

and therefore by (1.21)

$$
\begin{aligned}
\Omega_{\alpha \bar{\beta}}^{\prime \prime} & =\sum \omega_{\alpha \bar{i}} \wedge \omega_{i \bar{\beta}}=\sum \omega_{\alpha \bar{r}} \wedge \omega_{r \bar{\beta}}+\sum \omega_{\alpha \bar{\lambda}} \wedge \omega_{\lambda \bar{\beta}} \\
& =\Omega_{\alpha \bar{\beta}}+\sum\left(\bar{v}_{r \bar{\rho}} \omega_{\alpha \bar{\rho}}^{\prime}-\bar{v}_{r \bar{\lambda}} \omega_{\alpha \bar{\lambda}}\right) \wedge\left(v_{r \bar{\sigma}} \omega_{\sigma \bar{\beta}}^{\prime}-v_{r \bar{\mu}} \omega_{\mu \bar{\beta}}\right),
\end{aligned}
$$

establishing the first claim.

By Cramer's rule, each entry $v_{r \bar{\sigma}}$ of $\left(v_{r \bar{\sigma}}\right)=\left(u_{\rho \bar{s}}\right)^{-1}$ may be expressed as the quotient of a cofactor of $\left(u_{\rho \bar{s}}\right)$ by $\operatorname{det}\left(u_{\rho \bar{s}}\right)=\Delta$. Therefore the coefficients of the polynomial are $O\left(|\Delta|^{-2}\right)$, as required.

As a consequence, we infer from (4.14) that there is no problem with convergence when we integrate $\Psi$ over the fiber near points of nontransverse intersection. In particular, $c_{j}\left(\Omega^{\prime \prime}\right)$ involves a factor of $|\Delta|^{-2 j}, 0<j<k$, and thus by (4.14) integrating over the fiber involves a factor of $|\Delta|^{-2 j+2 k+2}$. Since the exponent is always positive, the integrals converge absolutely, in fact because of positivity of Chern forms. Therefore we may legitimately exclude points of nontransversality cavalierly from our integrals.

\section{Appendix.}

(a) Integration over the fiber and the Gysin map. In recent years, a great deal of machinery has been developed for dealing with integration over the fibers of, for example, a proper holomorphic mapping between complex analytic spaces. In quite general situations, this makes sense as a current (cf. [16], [26]), but our interest here is in smooth fiber bundles, where matters are much simpler (cf. [5]). We give here a brief summary of the necessary notation and relate the analytic process to the geometry of Poincaré duality and the Gysin maps, relationships which we exploit in $\$ \S 2$ and 3 . We begin our considerations with smooth manifolds and specialize to complex manifolds only at one point. 


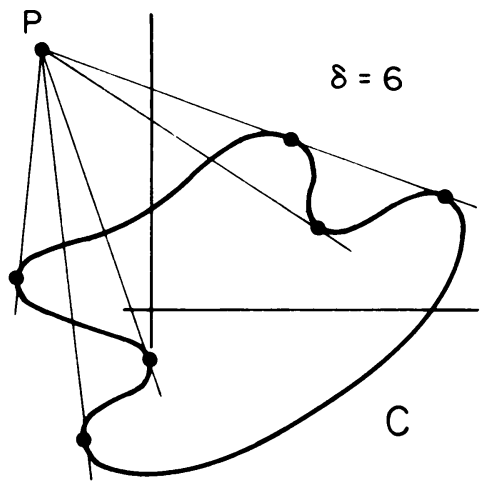

FIGURE 5

Let $F^{k} \stackrel{i}{\rightarrow} E^{m} \stackrel{\pi}{\rightarrow} B^{n}$ be a smooth fiber bundle, where $F, E$, and $B$ are compact, oriented smooth manifolds $(n+k=m)$. Given a complex-valued $(p+k)$-form $\phi$ on $E$, we define a $p$-form $\pi_{*} \phi$ on $B$ as follows. By using a partition of unity, we may suppose that supp $\phi \subset W$ (cf. Figure 5), where

(a) $W \subset \pi^{-1}(U), U$ lies in a coordinate neighborhood on $B$ and the bundle is trivial over $U$, and

(b) $i^{-1}(W) \subset V$, where $V$ lies in a coordinate neighborhood on $F$. Let $x^{1}, \ldots, x^{n}$ be base coordinates, and $y^{1}, \ldots, y^{k}$ be fiber coordinates on $W$. Write

$$
\begin{aligned}
\phi & =\sum_{|I|+|J|=p+k} \phi_{I J} d y^{J} \wedge d x^{I}, \text { where } \phi_{I J} \text { have compact support, } \\
& =\sum_{|I|=p} \phi_{I} d y^{1} \wedge \cdots \wedge d y^{k} \wedge d x^{I}+\sum_{|I|>p} \cdots
\end{aligned}
$$

Define

$$
\pi_{*} \phi=\sum_{|I|=p}\left(\int_{\pi^{-1}(x)} \phi_{I}(x, y) d y^{1} \wedge \cdots \wedge d y^{k}\right) d x^{I}
$$

One checks in a straightforward manner that $\pi_{*}$ is well defined, i.e., is independent of partition of unity and of coordinates. $\pi_{*}$ is integration over the fiber.

(A.1) Proposition. Let $\phi$ be $a(p+k)$-form on $E$, and let $\psi$ be an arbitrary $(n-p)$-form on $B$. Then $\int_{B} \pi_{*} \phi \wedge \psi=\int_{E} \phi \wedge \pi^{*} \psi$.

Thus $\pi_{*}$ is the adjoint operation to $\pi^{*}$. The proof of (A.1) is an easy application of Fubini's theorem and will be omitted.

(A.2) Corollary. $\pi_{*}: H_{D R}^{*}(E, \mathrm{C}) \rightarrow H_{D R}^{*}(B, \mathrm{C})$; i.e., $\pi_{*}$ maps closed forms to closed forms and exact forms to exact forms.

Proof. The fundamental fact is that $d$ and $\pi_{*}$ commute up to sign. We leave the verification of this to the reader.

The next step is to give a geometric interpretation of this procedure. The Gysin homomorphism $\pi_{!}$is defined to be the dual of $\pi_{*}: H_{*}(E, \mathrm{Z}) \rightarrow H_{*}(B, \mathrm{Z})$. That is, if 
we let $\mathscr{D}_{X}$ denote the Poincare duality isomorphism $\mathscr{D}_{X}: H_{k}(X, \mathbf{Z}) \stackrel{\approx}{\rightarrow} H^{n-k}(X, \mathbf{Z})$ on a compact, oriented $n$-dimensional manifold $X$, we define $\pi_{!}$by the commutative diagram:

$$
\begin{array}{ccc}
H^{p+k}(E, \mathbf{Z}) & \stackrel{\pi_{!}}{\rightarrow} & H^{p}(B, \mathbf{Z}) \\
\downarrow^{\mathscr{D}_{E}^{-1}} & & \uparrow \mathscr{D}_{B} \\
H_{m-p-k}(E, \mathbf{Z}) & \stackrel{\pi_{*}}{\rightarrow} & H_{m-p-k}(B, \mathbf{Z})=H_{n-p}(B, \mathbf{Z})
\end{array}
$$

Tensoring with $\mathbf{C}$, we have the same mapping on complex cohomology, which by DeRham theory we may represent by differential forms; we claim that $\pi_{!}$: $H^{p+k}(E, \mathrm{C}) \rightarrow H^{p}(B, \mathrm{C})$ is integration over the fiber.

The following basic equation may be taken as the definition of Poincare duality (cf. [24]).

For $\phi \in H^{k}(X, \mathbf{Z})$ and $\sigma \in H_{k}(X, \mathbf{Z}), \phi(\sigma)=\left(\mathscr{D}_{X} \cup \phi\right)([X])$, where $[X]$ is the fundamental homology class.

In terms of DeRham theory, if $Z$ is an oriented $k$-dimensional submanifold and $\phi$ a closed $k$-form,

$$
\int_{Z} \phi=\int_{X} \mathscr{D}_{X}([Z]) \wedge \phi
$$

Now, to see that $\pi_{*}$ and $\pi_{\text {! }}$ agree, let $\phi$ be a closed $(p+k)$-form representing the cohomology class $[\phi] \in H^{p+k}(E, C)$, and let $\psi$ be a closed $(n-p)$-form on $B$. Following the diagram and applying (A.4) and then (A.1), we find

$$
\begin{aligned}
\int_{B} \pi_{!} \phi \wedge \psi & =\int_{B}\left(\mathscr{D}_{B} \pi_{*} \mathscr{D}_{E}^{-1} \phi\right) \wedge \psi=\int_{\pi_{*} \mathscr{D}_{E}^{-1} \phi} \psi=\int_{\mathscr{D}_{E}^{-1} \phi} \pi^{*} \psi \\
& =\int_{E} \phi \wedge \pi^{*} \psi=\int_{B} \pi_{*} \phi \wedge \psi,
\end{aligned}
$$

as required.

In the event that the fiber bundle is a holomorphic fiber bundle, we can be a little more explicit. If $\phi$ is a $(p+k, q+k)$-form on $E$ and the fiber is a $k$-dimensional compact complex manifold, then $\pi_{*} \phi$ is a $(p, q)$-form on $B$. This is immediate from either definition.

Lastly we would like to comment on invariant theory vis-à-vis integration over the fiber. Suppose $E \rightarrow B$ is a fiber bundle and $G$ acts equivariantly on $E$ (but not necessarily transitively on $B$ ). Then if $\phi$ is an invariant form on $E, \pi_{*} \phi$ is an invariant form on $B$. This is an immediate consequence of the more general

LEMmA. Let $E \stackrel{\pi}{\rightarrow} B, E^{\prime} \stackrel{\pi^{\prime}}{\rightarrow} B^{\prime}$ be fiber bundles such that there are orientation-preserving diffeomorphisms $f: E \rightarrow E^{\prime}, g: B \rightarrow B^{\prime}$ with $\pi^{\prime} \circ f=g \circ \pi$. Then $\pi_{*} f^{*}=$ $g^{*} \pi_{*}^{\prime}$. 
Proof. Let $\phi$ be a $(p+k)$-form on $E^{\prime}, \psi$ an arbitrary $(n-p)$-form on $B^{\prime}$ ( $k=$ dimension fiber). Then

$$
\begin{aligned}
\int_{B} \pi_{*} f^{*} \phi \wedge g^{*} \psi & =\int_{E} f^{*} \phi \wedge \pi^{*} g^{*} \psi=\int_{E} f^{*} \phi \wedge f^{*} \pi^{*} \psi=\int_{E^{\prime}} \phi \wedge \pi^{* *} \psi \\
& =\int_{B^{\prime}} \pi_{*}^{\prime} \phi \wedge \psi=\int_{B} g^{*} \pi_{*}^{\prime} \phi \wedge g^{*} \psi
\end{aligned}
$$

Since $\psi$ is arbitrary and $g$ is a diffeomorphism, $\pi_{*} f^{*} \phi=g^{*} \pi_{*}^{\prime} \phi$, as required.

(b) Invariant theory and the cohomology of flag spaces. Using Hermann Weyl's theorem (1.27) we now provide proofs of Theorems (1.28) and (3.7) upon which our invariant theoretic considerations are based. The proof is essentially that given by Chern [3] in his original definition of Chern classes.

(1.28) THEOREM. The invariant forms on $G(p, n)$ are polynomials with constant coefficients in the Chern forms $c_{i}\left(\Omega_{E}\right)$ of the universal subbundle. Hence the Chern classes $c_{i}(E)$ generate $H^{*}(G(p, n), \mathbf{R})$.

Proof. Recall $G(p, n) \cong U(n) / U(p) \times U(n-p)=G / H$. Since $G$ acts transitively on $G / H$, it suffices to fix a point $z_{0}$ and compute the forms on $T_{z_{0}} G(p, n)$ invariant under the action of the isotropy subgroup $H$. Thus we seek a polynomial with constant coefficients in $\omega_{\alpha \bar{\mu}}, \bar{\omega}_{\alpha \bar{\mu}}$ which are invariant. Let

$$
h=\left(\begin{array}{c|c}
h_{\alpha \bar{\beta}} & 0 \\
\hline 0 & h_{\mu \bar{\nu}}
\end{array}\right) \in H ;
$$

put $\omega_{\alpha \bar{\mu}}^{\prime}=L_{h}^{*}\left(\omega_{\alpha \bar{\mu}}\right)=\sum h_{\alpha \bar{\beta}} \bar{h}_{\mu \bar{\nu}} \omega_{\beta \bar{\nu}}$. The proof proceeds in two steps which we state as separate lemmas.

(A.5) Lemma. Any polynomial in $\omega_{\alpha \bar{\mu}}, \bar{\omega}_{\alpha \bar{\mu}}$ which is invariant under the action of $U(n-p)$ is a polynomial in the curvature form $\Omega_{\alpha \bar{\beta}}=-\Sigma \omega_{\alpha \bar{\mu}} \wedge \bar{\omega}_{\beta \bar{\mu}}$.

(A.6) LemmA. A polynomial in $\Omega_{\alpha \bar{\beta}}$ which is invariant under the left action or adjoint action (1.8) of $U(p)$ is a polynomial with constant coefficients in the Chern forms $c_{i}\left(\Omega_{\alpha \bar{\beta}}\right)$.

Proof of Lemma (A.5). Write $T_{z_{0}} G(p, n)=W$ as $\mathbf{C}^{p *} \otimes \mathbf{C}^{n-p}$ with basis $e^{\alpha} \otimes$ $e_{\mu}$. A typical vector $w \in W$ may be written as $w=\Sigma w_{\alpha \bar{\mu}} e^{\alpha} \otimes e_{\mu}$, and the action of $U(n-p)$ on $W$ is given by

$$
\left(e^{\alpha} \otimes e_{\mu}\right)^{\prime}=\sum h_{\mu \bar{\nu}}\left(e^{\alpha} \otimes e_{\nu}\right)
$$

Let $\psi$ be a $(k, l)$-form on $W$ which is invariant under the $U(n-p)$ action, and view $\psi$ as an alternating $(k+l)$-tensor. Fix $\alpha_{1}, \ldots, \alpha_{k}, \beta_{1}, \ldots, \beta_{l}$ and let $\psi_{1}$ be the collection of all terms in $\psi$ of the form

$$
\omega_{\alpha_{1} \bar{\mu}_{1}} \wedge \cdots \wedge \omega_{\alpha_{k} \bar{\mu}_{k}} \wedge \bar{\omega}_{\beta_{1} \bar{\nu}_{1}} \wedge \cdots \wedge \bar{\omega}_{\beta_{l} \bar{\nu}_{i}}
$$

Let $w_{1}, \ldots, w_{k+l}$ be arbitrary vectors in $W$, and put $w_{i}=\sum w_{i, \alpha \bar{\mu}} e^{\alpha} \otimes e_{\mu}$. Applying $\psi_{1}$ to $\left(w_{1}, \ldots, w_{k+l}\right)$ will give an invariant function of the components of the 
vectors

$$
\left.\begin{array}{l}
v_{i, \alpha_{j}}=\sum w_{i, \alpha_{j} \bar{\mu}} e_{\mu}, \quad j=1, \ldots, k \\
v_{i, \beta_{m}}=\sum w_{i, \beta_{m} \bar{\mu}} e_{\mu}, \quad m=1, \ldots, l
\end{array}\right\}, \quad i=1, \ldots, k+l,
$$

and their conjugates. Invariance under the $U(n-p)$ action implies that this must be an expression in inner products of these various vectors. Since

$$
\left(v_{i, \alpha_{j}}, v_{h, \beta_{m}}\right)=\sum w_{i, \alpha_{j} \bar{\mu}} \bar{w}_{h, \beta_{m} \bar{\mu}}=\left(\sum \omega_{\alpha, \bar{\mu}} \otimes \bar{\omega}_{\beta_{m} \bar{\mu}}\right)\left(v_{i}, v_{h}\right),
$$

and so on, we conclude that $\psi_{1}$ must be a polynomial in $\Sigma \omega_{\alpha_{j} \bar{\mu}} \wedge \bar{\omega}_{\beta_{m} \bar{\mu}}$; therefore, $\psi$ is a polynomial in $\Sigma \omega_{\alpha \bar{\mu}} \wedge \bar{\omega}_{\beta \bar{\mu}}$, as required. (Note that other terms appear when we take inner products, such as $\left(v_{i, \alpha_{j}}, v_{h, \alpha_{m}}\right)$; this corresponds to the form $\Sigma \omega_{\alpha, \bar{\mu}} \wedge \bar{\omega}_{\alpha_{m} \bar{\mu}}$ and hence appears in the form $\psi_{1}$ if and only if $\alpha_{m}=\beta_{m^{\prime}}$ for some $m^{\prime}$.)

Proof of Lemma (A.6). Step 1. The first part of the proof consists in proving that an invariant form in $\Omega_{\alpha \bar{\beta}}$ must be a polynomial in the forms

$$
\Phi_{i}=\sum \Omega_{\alpha_{1} \bar{\alpha}_{2}} \wedge \Omega_{\alpha_{3} \bar{\alpha}_{4}} \wedge \cdots \wedge \Omega_{\alpha_{i-1}}, \quad i=1, \ldots, p .
$$

The procedure is similar to that used in the proof of Lemma (A.5) and will only be sketched here. It is elementary to check that an invariant 2-form can only be a multiple of $\Sigma \Omega_{\alpha \bar{\alpha}}(=\operatorname{tr}(\Omega))$.

Given an invariant $2 q$-form $\psi$ which is a polynomial in $\Omega_{\alpha \bar{\beta}}$, and arbitrary vectors $w_{1}, \ldots, w_{2 q} \in W$, define

$$
v_{\alpha}^{\{i, j\}}=\sum_{\beta}\left(\sum_{\mu} w_{i, \alpha \bar{\mu}} \bar{w}_{j, \beta \bar{\mu}}\right) e_{\beta} \in \mathbf{C}^{p},
$$

and, in general,

$$
v_{\alpha}^{\left\{i_{1}, \ldots, i_{2 q}\right\}}=\sum_{\beta}\left(\sum w_{i_{1} \alpha \bar{\mu}_{1}} \bar{w}_{i_{2} \beta_{1} \bar{\mu}_{1}} \cdots w_{i_{2 q-1} \beta_{q-1} \bar{\mu}_{q}} \bar{w}_{i_{2 q} \beta \bar{\mu}_{q}}\right) e_{\beta} .
$$

Note that $v_{\alpha \beta}^{\{i, j\}}=-\Omega_{\alpha \bar{\beta}}\left(w_{i}, w_{j}\right)$, and so $\psi\left(w_{1}, \ldots, w_{2 q}\right)$ is expressible in terms of all $v_{\alpha \beta}^{\{i, j\}}$. On the other hand, by (1.27) this must be a polynomial in inner products

$$
\left(v_{\alpha_{1}}^{\left\{i_{1}, j_{1}\right\}}, v_{\alpha_{2}}^{\left\{i_{2}, j_{2}\right\}}\right)=\sum w_{i_{1} \alpha_{1} \bar{\mu}_{1}} \bar{w}_{j_{1} \beta \bar{\mu}_{1}} \bar{w}_{i_{2} \alpha_{2} \bar{\mu}_{2}} w_{j_{2} \beta \bar{\mu}_{2}}
$$

but this is the $\alpha_{2}$-component of the vector $v_{\alpha_{1}}^{\left\{i_{1}, j_{1}, i_{2}, j_{2}\right\}}$, and so forth. On the other hand, skew-symmetrizing, the inner product is given by $\sum \Omega_{\alpha_{1} \bar{\beta}} \wedge$ $\Omega_{\beta \bar{\alpha}_{2}}\left(w_{i_{1}}, w_{j_{1}}, w_{i_{2}}, w_{j_{2}}\right)$. So we can instead formally introduce vectors of forms as follows:

$$
\begin{aligned}
& \Omega_{\alpha \bar{\beta}}^{(i)}=\sum \Omega_{\alpha \bar{\beta}_{1}} \wedge \Omega_{\beta_{1} \bar{\beta}_{2}} \wedge \cdots \wedge \Omega_{\beta_{i-1} \bar{\beta}}, \quad \Omega_{\alpha \bar{\beta}}^{(1)}=\Omega_{\alpha \bar{\beta}}, \\
& \Omega_{\alpha}^{(i)}=\sum \Omega_{\alpha \bar{\beta}}^{(i)} \otimes e_{\beta}, \quad \Omega^{(i)}=\sum \Omega_{\alpha}^{(i)} \otimes e_{\bar{\alpha}} .
\end{aligned}
$$

Then it is not hard to check that $\psi$ is a polynomial in "inner products" $\left(\Omega_{\alpha}^{(i)}, \Omega_{\beta}^{(j)}\right)=$ $(-1)^{j} \Omega_{\alpha \beta}^{(i+j)}$, and hence in $\left(\Omega^{(i)}, \Omega^{(j)}\right)=(-1)^{j} \Phi_{i+j}$, as required. This amounts to a descending induction on the number of unpaired indices.

Step 2. Next one must verify that the forms $\Phi_{i}$ are polynomials in the Chern forms $c_{j}(\Omega)$. Clearly $c_{1}(\Omega)$ and $\Phi_{1}$ differ only by a constant. Proceeding by induction, we examine the formula $(1.9)$ for $c_{j}(\Omega)$ more closely. 


$$
\begin{aligned}
c_{j}(\Omega) & =\left(\frac{\sqrt{-1}}{2 \pi}\right)^{j} \frac{1}{j !} \sum_{\sigma} \operatorname{sgn} \sigma \Omega_{\alpha_{1} \bar{\alpha}_{\sigma(1)}} \wedge \cdots \wedge \Omega_{\alpha_{j}, \bar{\alpha}_{\alpha()}} \\
& =\left(\frac{\sqrt{-1}}{2 \pi}\right)^{j} \frac{1}{j !}\left(\Sigma_{1}+\Sigma_{2}\right),
\end{aligned}
$$

where $\Sigma_{1}$ is the sum over all $\sigma \in S_{j}$ with no fixed point and $\Sigma_{2}$ is the sum over all $\sigma \in S_{j}$ with a fixed point. The second term involves terms of the form

$$
\sum_{\sigma^{\prime} \in S_{j-1}} \Omega_{\alpha \bar{\alpha}} \wedge \Omega_{\alpha_{1} \bar{\alpha}_{\sigma^{\prime}(1)}} \wedge \cdots \wedge \Omega_{\alpha_{j-1} \alpha_{\sigma^{\prime}(j-1)}}
$$

which by induction can be expressed as a polynomial in $\Phi_{1}, \ldots, \Phi_{j-1}$. On the other hand, all the permutations appearing in the first sum are cycles of full length $j$ and hence all have $\operatorname{sgn} \sigma=(-1)^{j}$, and this expression differs from $\Phi_{j}$ only by a constant. Thus the proof of the lemma is complete.

From these two lemmas the theorem follows immediately. The statement about cohomology follows from (1.26). We now turn to the proof of the

(3.7) THEOREM. Modulo the differential ideal $\mathcal{G}=\left(\omega^{\mu}, \omega^{\bar{\mu}}\right)$, any invariant form on $\mathbf{G}(0, p, n)$ is a polynomial with constant coefficients in the (truncated) Kähler form $\omega=\sum \omega^{\alpha} \wedge \omega^{\bar{\alpha}}$ and the Chern forms $c_{k}\left(\Omega_{i j}\right)$ of the universal bundle $E_{p+1}$ on $\mathbf{G}(0, p, n)$.

Proof. Since $\mathbf{G}(0, p, n) \cong U(n+1) /(U(1) \times U(p) \times U(n-p))$ is homogeneous, any invariant form is determined by its values at a fixed point $\left(z_{0}, T_{0}\right)$, and therefore, as before, the computation reduces to finding expressions in the Maurer-Cartan forms of $U(n+1)$ which are left-invariant under the subgroup $H=U(1) \times U(p) \times U(n-p)$.

The proof follows the same lines as that of Theorem (1.28) just given. At this point, we give the invariant theoretical arguments in a more formal fashion; they may nevertheless be reduced to Theorem (1.27) in the same way as before. We define formal vectors as follows:

$$
\begin{gathered}
\psi=\sum \omega^{\alpha} \otimes e_{\alpha}, \quad \psi_{\alpha}=\sum \omega_{\alpha \bar{\mu}} \otimes e_{\mu}, \quad \psi_{\bar{\mu}}=\sum \omega_{\alpha \bar{\mu}} \otimes e^{\alpha}, \\
\Omega^{(i)}=\sum \Omega_{\alpha \beta}^{(i)} \otimes e_{\beta} \quad\left(\Omega_{\alpha \bar{\beta}}^{(i)}=\Omega_{\alpha \bar{\beta}} \wedge \cdots \wedge \Omega_{\beta_{i-1} \bar{\beta}}\right), \\
\Omega^{(i)}=\sum \Omega_{\alpha}^{(i)} \otimes e^{\alpha} .
\end{gathered}
$$

Note that $U(1)$-invariance implies that $\psi$ must appear paired with $\bar{\psi}$. As in the earlier argument, $U(n-p)$-invariance implies that the components of $\psi_{\alpha}$ are paired in the curvature form $\Omega_{\alpha \bar{\beta}}=-\sum \omega_{\alpha \bar{\mu}} \wedge \bar{\omega}_{\beta \bar{\mu}}$. Lastly we exploit invariance under the action of $U(p)$. As before, we get terms $\left(\Omega^{(i)}, \Omega^{(j)}\right)$ which are essentially Chern forms; we get in addition $(\psi, \psi)=\Sigma \omega^{\alpha} \wedge \omega^{\bar{\alpha}}=\omega,\left(\Omega_{\alpha}^{(i)}, \psi\right)$ and $\left(\Omega^{(i)}, \psi\right)$. However, terms of the latter sort vanish modulo $g$ since $\sum \omega^{\alpha} \wedge \omega_{\alpha \bar{\mu}}=d \omega^{\mu} \equiv 0$.

Therefore, the invariant forms are polynomials in Chern forms $c_{k}\left(\Omega_{\alpha \bar{\beta}}\right)$ and the Kähler form $\omega$. It remains only to observe that the forms $c_{k}\left(\Omega_{\alpha \bar{\beta}}\right)$ may be expressed in terms of $c_{k}\left(\Omega_{i j}\right)$ and the Kähler form, insofar as $\left(\Omega_{\alpha \bar{\beta}}\right)$ is the curvature matrix of the quotient bundle $E_{p+1} / H^{-1}$ (cf. (1.10)). 
We remark that all the invariant forms which appear in the conclusions of this theorem are closed; what is not so clear is that these forms generate all the cohomology of the pointed Grassmannian $\mathbf{G}(0, p, n)$. Rather than checking explicitly that the remaining invariant forms are not closed, we resort to a quick argument using spectral sequences.

Proposition. Let $1 \leqslant k<l<n$. Then $H^{*}(G(k, l, n), \mathbf{Z}) \cong H^{*}(G(k, l), \mathbf{Z}) \otimes$ $H^{*}(G(l, n), \mathbf{Z})$.

Proof. The main point is that $G(k, l, n)$ fibers over $G(l, n)$ with fiber $G(k, l)$ and that the cohomology algebra $H^{*}(G(m, n), \mathbf{Z})$ has elements of only even degree.

In general, given a fiber bundle $F \rightarrow E \rightarrow B$ with, for example, $\pi_{1}(B)=0$, there is a spectral sequence [22, p. 80], [24, Chapter 9] $E_{r}^{p q} \Rightarrow E_{\infty}^{p q}$ with

(i) $E_{1}^{p q}=C^{p}\left(B, H^{q}(F, \mathbf{Z})\right)$ and $E_{2}^{p q}=H^{p}\left(B, H^{q}(F, \mathbf{Z})\right)$,

(ii) $H^{m}(E, \mathbf{Z}) \cong \bigoplus_{p+q=m} E_{\infty}^{p q}$.

In our case, since both fiber and base space have no cohomology in odd degree, we have the situation pictured in Figure 6. $d_{2}: E_{2} \rightarrow E_{2}$ is of bidegree $(+2,-1)$ and is therefore clearly the zero map. Therefore the spectral sequence degenerates at the $E_{2}$ term and $E_{\infty}=E_{2}$. Thus, $H^{m}(G(k, l, n), \mathbf{Z}) \simeq \bigoplus_{p+q-m} H^{p}(G(l, n), \mathbf{Z}) \otimes$ $H^{q}(G(k, l), \mathbf{Z})$ by the universal coefficient theorem. This proves the proposition.

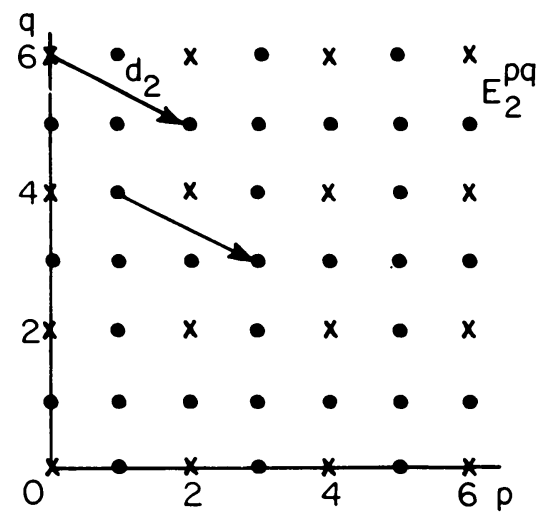

FIGURE 6

COROllaRY. $H^{*}(\mathbf{G}(0, p, n), \mathbf{Z})$ is generated as an algebra by the class of the truncated Kähler form $\omega$ and the Chern classes of the universal bundle on $\mathbf{G}(p, n)$.

\section{BIBLIOGRAPHY}

1. E. Cartan, Sur les invariants intégraux de certains espaces homogènes clos et les propriétés topologiques de ces espaces, Ann. Soc. Polon. Math. 8 (1929), 181-225.

2. S. S. Chern, On integral geometry in Klein spaces, Ann. of Math. (2) 43 (1942), 178-189.

3. C__ Characteristic classes of hermitian manifolds, Ann. of Math. (2) 47 (1946), 85-121.

4. $\ldots$ On the kinematic formula in the euclidean space of $n$ dimensions, Amer. J. Math. 74 (1952), $227-236$.

5. On the characteristic classes of complex sphere bundles and algebraic varieties, Amer. J. Math. 75 (1953), 565-597.

6. __ On the kinematic formula in integral geometry, J. Math. Mech. 16 (1966), 101-118.

7. __ Complex manifolds without potential theory, Springer-Verlag, Berlin and New York, 1979. 
8. S. S. Chern and P. A. Griffiths, Abel's theorem and webs, Jahresber. Deutsch. Math.-Verein. 80 (1978), 13-110.

9. F. J. Flaherty, The volume of a tube in complex projective space, Illinois J. Math. 16 (1972), 627-638.

10. P. A. Griffiths, Hermitian differential geometry, Chern classes, and positive vector bundles, Global Analysis (papers in honor of K. Kodaira), Princeton Univ. Press, Princeton, N.J., 1969, pp. 185-251.

11. , Entire holomorphic mappings in one and several complex variables, Princeton Univ. Press, Princeton, N.J., 1976.

12. , Complex differential and integral geometry and curvature integrals associated to singularities of complex analytic varieties, Duke Math. J. 45 (1978), 427-512.

13. P. A. Griffiths and M. Cornalba, Some transcendental aspects of algebraic geometry, Proc. Sympos. Pure Math. vol. 29, Amer. Math. Soc., Providence, R.I., 1975, pp. 3-110.

14. P. A. Griffiths and J. Harris, Principles of algebraic geometry, Wiley, New York, 1978.

15. F. Hirzebruch, Topological methods in algebraic geometry, Die Grundlehren der Math. Wissenschaften, Band 131, Springer-Verlag, New York, 1966.

16. J. King, Currents defined by analytic varieties, Acta Math. 127 (1971), 185-220.

17. S. Kobayashi and K. Nomizu, Foundations of differential geometry, Wiley, New York, 1963 and Interscience, New York, 1969.

18. R. Langevin, Courbure et singularités complexes, Comment. Math. Helv. 54 (1979), 6-16.

19. R. Langevin and T. Shifrin, Polar varieties and integral geometry (to appear).

20. R. MacPherson, Chern classes for singular algebraic varieties, Ann. of Math. (2) 100 (1974), 423-432.

21. J. Milnor and J. Stasheff, Characteristic classes, Princeton Univ. Press, Princeton, N.J., 1974.

22. R. Mosher and M. Tangora, Cohomology operations and applications in homotopy theory, Harper and Row, New York, 1968.

23. L. A. Santaló, Integral geometry and geometric probability, Addison-Wesley, Reading, Mass., 1976.

24. E. H. Spanier, Algebraic topology, McGraw-Hill, New York, 1966.

25. M. Spivak, A comprehensive introduction to differential geometry, Vol. V, Publish or Perish, Boston, Mass., 1975.

26. W. Stoll, Value distribution of holomorphic maps into compact complex manifolds, Lecture Notes in Math., vol. 135, Springer-Verlag, Berlin and New York, 1970.

27. __ Invariant forms on Grassmann manifolds, Princeton Univ. Press, Princeton, N.J., 1977.

28. R. O. Wells, Differential analysis on complex manifolds, Springer-Verlag, New York, 1980.

29. H. Weyl, On the volume of tubes, Amer. J. Math. 61 (1939), 461-472.

30. __ The classical groups, Princeton Univ. Press, Princeton, N.J., 1953.

Department of Mathematics, Massachusetts institute of Technology, Cambridge, MasSACHUSETTS 02139 